\title{
Shortfall risk minimization in discrete time financial market models
}

\author{
Noufel Frikha*
}

January 31, 2012

\begin{abstract}
In this paper, we study theoretical and computational aspects of risk minimization in financial market models operating in discrete time. To define the risk, we consider a class of convex risk measures defined on $L^{p}(\mathbb{P})$ in terms of shortfall risk. Under simple assumptions, namely the absence of arbitrage opportunity and the non-degeneracy of the price process, we prove the existence of an optimal strategy by performing a dynamic programming argument in a nonMarkovian framework. Optimal strategies are shown to satisfy a first order condition involving the constructed Bellman functions. In a Markovian framework, we propose and analyze several algorithms based on Monte Carlo simulations to estimate the shortfall risk and optimal dynamic strategies. Finally, we illustrate our approach by considering several shortfall risk measures and portfolios inspired by energy and financial markets.
\end{abstract}

Keywords: Convex risk measure, shortfall risk measures, risk minimization, dynamic programming principle, stochastic approximation algorithm, vector quantization, Monte Carlo simulation.

\section{Introduction}

In an arbitrage-free and complete financial market model, an investor endowed with a contingent claim can remove its risk perfectly by using a self-financing strategy on finite horizon time. The unique cost of replication is the expected value of the contingent claim under the unique equivalent martingale measure. However, in a more realistic point of view, market models are incomplete. The no-arbitrage assumption no longer provides a unique equivalent pricing martingale measure. Hence it leads to an interval of arbitrage-free prices. Pricing and hedging in such context require new methods. It is still possible to hedge without any risk whenever an agent invests a large enough initial wealth and uses a super-hedging criterion, see e.g. [15], 28], 44] and [18] among others. However, the price is often excessively high since the super-hedging strategy reduces to the trivial buy-and-hold strategy, see e.g. [14], [5] and [8]. For this reason, an investor faced with a contingent claim can only hedge partially and often has to bear some risk of loss.

Numerous criteria have been proposed to quantify this risk. Hedging by means of a quadratic criteria consists in minimizing the $L^{2}(\mathbb{P})$-norm of the difference between the value of the contingent claim and the terminal value of a self-financing portfolio. This approach has been proposed in the seminal paper [11] and then studied in [43, [45, [46, [32, 38, [30, among others. The main criticism opposed to this approach is that the $L^{2}(\mathbb{P})$-norm is a symetric criteria. Hence, it gives equal importance to upside and downside values. Hedging strategies which maximize the probability of successfull hedge without risk are investigated in [19] in a general continuous time semimartingale model as an alternative to super-hedging strategy which requires a large amount of initial capital. However, this approach is known to take into account only the probability of a successfull hedge and not the size of effective losses when they occur.

${ }^{*}$ CMAP, Ecole Polytechnique Paris, CNRS, e-mail: noufel.frikha@cmap.polytechnique.fr 
In order to take into account the importance of downside values and hence to overcome the symmetry introduced by a quadratic hedging, several authors proposed to use an asymmetric criteria in both discrete and continuous time models. For instance in [7], the authors investigated the following dynamic measures for the risk associated to the contingent claim $H$

$$
\sup _{\mathbb{Q} \in \mathcal{D}} \inf _{\theta \in \mathcal{A}} \mathbb{E}_{\mathbb{Q}}\left[\left(H-V_{T}^{x, \theta}\right)_{+}\right]
$$

where $\mathcal{D}$ is a suitable family of probability measures, $V_{T}^{x, \theta}$ is the terminal value of the portfolio according to the self-financing strategy $\theta$ with initial capital $x$ and $\mathcal{A}$ is a specific class of admissible portfolio strategies. In particular, the wealth process $\left(V_{t}^{x, \theta}\right)_{t \in[0, T]}$ has to satisfy a lower bound condition. In [35, the author studies the problem of minimizing the expected $L^{p}$-loss, $p \in(1,+\infty)$, of the shortfall of a contingent claim $H \in L^{p}(\mathbb{P})$ in a general discrete time financial market model:

$$
\inf _{\theta \in \mathcal{A}} \mathbb{E}\left[l_{p}\left(\left(H-V_{T}^{x, \theta}\right)_{+}\right)\right],
$$

where $l_{p}(x)=x^{p} / p, x \geq 0$, under cone constraints on trading strategies. In a general semimartingale setting, this problem has been solved in 20] for a general loss function $l$ using an approach based on Neyman-pearson lemma.

In this paper, we consider the problem of risk minimization in a general discrete time financial market model by means of a shortfall risk based convex risk measures criteria defined on $L^{p}(\mathbb{P})$ (see below for this notion). The seminal axioms for coherent risk measures were introduced in [1] and then were further generalized in [21] and [23] to define convex risk measures. However, these risk measures were only defined for bounded financial positions. This is too restrictive for applications like stochastic control problem involving risk measures. Moreover, as argued in [17] for instance, it is natural to consider coherent and convex risk measures in the space $L^{p}(\mathbb{P})$ which carries a locally convex topology.

Surprisingly, despite its pratical importance, it seems that risk minimization in discrete time financial market models has been somewhat neglected both from a theoretical and numerical point of view. In [4], the authors developped a risk minimization problem to hedge non-tradable risk on financial market using an approach based on inf-convolution of convex risk measures and BSDE. In [39], a portfolio optimization method which calculates the Value-at-Risk at level $\alpha\left(\operatorname{VaR}_{\alpha}\right)$ and optimizes the Conditional Value-at-Risk $\left(\mathrm{CVaR}_{\alpha}\right)$ is developped. The numerical procedure is based on a linear programming algorithm. It first consists in generating loss scenarios and then in introducing them as constraints in the linear programming problem. The main drawback is that the dimension (number of constraints) of the linear programming problem to be solved is equal to the number of simulated scenarios so that this approach turns out to have strong limitations in practice. Moreover, the authors only focused on static portfolio optimization. The framework they proposed doesn't take into account self-financed strategies.

In [3, the authors considered the problem of risk minimization to hedge observable but nontradable source of risk by means of a CVaR criteria. In order to prove the existence of an optimal dynamic strategy for the CVaR minimization problem, a non Markovian dynamic programming principle is established (see e.g. [16]). The arguments given in [3] relies fundamentally on the Rockafellar \& Uryasev's static representation of the CVaR as a convex optimization problem. The optimal strategy is computed by minimizing dynamically the CVaR using three main numerical probabilistic tools: stochastic approximation algorithm, optimal quantization and variance reduction techniques. From a numerical point of view, the approach developed in [3] relies on devising time-consistent risk measures closely connected to the CVaR, based on a new conditional risk measure (called the $\mathcal{F}$-CVaR, where $\mathcal{F}$ is a $\sigma$-field representing the information available to investors), for which efficient and robust optimization procedures can be designed. 
Concerning the risk minimization problem by means of a shortfall risk measure defined on $L^{p}(\mathbb{P})$, such arguments do not work. Roughly speaking, this is due to the fact that a shortfall risk measure does not write as an expectation contrary to the CVaR but appears as the level of a function which can be written as the expectation of a loss (see below for more details). This induces theoretical and computational difficulties. However, one of the main contribution of this paper is to prove that a direct stochastic control approach in two steps, one of which consisting in a dynamic programming argument, is still possible. Unlike [3], we take full advantage of the dynamic programming backward induction to deduce first order conditions and use them to derive a completely tractable algorithm relying on stochastic approximation algorithm and optimal quantization. Let us mention that a dynamic programming principle in a non-Markovian setting has already been established in the context of expected utility maximization in discrete time models in [37.

Hence, the contribution of the paper is twofold.

Firstly, our main focus is theoretical. We prove the existence of optimal strategies for the shortfall risk minimization problem under simple assumptions by performing a dynamic programming argument in a non Markovian framework. Moreover, we characterize optimal strategies by showing that they satisfy first order conditions writing as a backward induction. As it will become clear in the arguments given below, these first order conditions are crucial for devising our numerical procedures.

Secondly, we are concerned by computational and numerical aspects of shortfall risk minimization. We propose robust and tractable numerical procedures for estimating the quantities of interest and analyze their properties. The first one is obviously the shortfall risk of a risky position. In order to estimate this quantity, we propose a stochastic approximation algorithm. We study its a.s. convergence and convergence rate. Then, we take advantage of the first order dynamic programming conditions to propose a procedure based on optimal vector quantization and stochastic gradient algorithm for estimating optimal dynamic strategies. One interesting consequence of our methodology may lie in the computation of shortfall risk based risk indifference prices of a contingent claim.

The paper is organized as follows: in Section 2, we present the model, definitions and the properties of shortfall risk measures. We outline the precise formulation of the risk minimization problem and we prove the existence of an optimal strategy that minimizes the shortfall risk under simple assumptions namely the absence of arbitrage opportunity and the non-degeneracy of the underlying price process. We characterize optimal strategies by proving, under some regularity assumptions, that they satisfy first order conditions involving the constructed Bellman functions. Section 3 is devoted to computational and numerical aspects of shortfall risk minimization. We propose a Robbins-Monro algorithm to estimate the risk of a financial position. We establish its a.s. convergence and convergence rate. We show how to devise a Monte Carlo simulation based NewtonRaphson's optimization algorithm to compute optimal one step self-financed strategies, that are strategies where the holder of the portfolio is allowed to adjust his portfolio only once at time $t=0$. Finally, we present our algorithm to comute optimal dynamic self-financed strategies that minimize the shortfall risk. In order to approximate conditional expectations and portfolio values at each time step, we rely on optimal vector quantization. Section 4 is dedicated to numerical examples. We propose several portfolios with several shortfall risk measures and illustrate the effectiveness of the proposed methodology. 


\section{Theoretical aspects of shortfall risk hedging}

\subsection{Problem formulation}

The discrete time and finite horizon financial market model is described as follows. We consider a filtered probability space $\left(\Omega, \mathcal{F},\left(\mathcal{F}_{t}\right)_{0 \leq t \leq N}, \mathbb{P}\right)$, where $\mathcal{F}_{N}=\mathcal{F}$ and for convenience we set $\mathcal{F}_{0}=$ $\{\varnothing, \Omega\}$. We have $d$ risky assets available for trade with (discounted) price process $S=\left(S^{1}, \cdots, S^{d}\right)$, with for all $t, S_{t}^{i} \geq 0, i=1, \cdots, d$ and $S$ is $\mathcal{F}$-adapted, i.e. $S_{t}$ is $\mathcal{F}_{t}$-measurable. A trading strategy (or portfolio) is an $\mathbb{R}^{d}$-valued process $\theta=\left(\theta_{t}^{1}, \cdots, \theta_{t}^{d}\right)_{1 \leq t \leq N}$ which is $\mathcal{F}$-predictable, i.e., $\theta_{t}$ is $\mathcal{F}_{t-1^{-}}$ measurable, $t=1, \cdots, N$. In such a strategy, we may regard $\theta_{t}$ as the number of units of risky assets held by the investor during $(t-1, t], t=1, \cdots, N$. The value at time $t$ of a (self-financed) portfolio $\theta$ starting from initial capital $v$ is given by $V_{0}^{v, \theta}=v$ and for $t=1, \cdots, N$,

$$
V_{t}^{v, \theta}=v+\sum_{\ell=1}^{t} \theta_{\ell} \cdot \Delta S_{\ell},
$$

where $\Delta S_{\ell}=S_{\ell}-S_{\ell-1}$. For $x, y \in \mathbb{R}^{d}$, the real number $x . y$ stands for their usual scalar product and $|x|:=\sqrt{x \cdot x}$ denotes the Euclidean norm of $x$. In what follows, we denote by $\Theta_{t}$ the space of all $\mathcal{F}_{t}$-measurable and $\mathbb{P}$-a.s. finite random variables with values in $\mathbb{R}^{d}$. Note that in contrast to the case of continuous time models, we don't require admissibility condition on strategies like the lower boundedness of the portfolio values.

We assume that the holder of the portfolio faced with a contingent claim given by an $\mathcal{F}_{N^{-}}$ measurable random variable $H \in L^{p}(\mathbb{P})$ assesses his risk using a static $L^{p}$ shortfall risk measure $\rho$. It is a classical example of convex risk measure, see [21], [22] for the $L^{\infty}(\mathbb{P})$ case and [26], [17] for the $L^{p}(\mathbb{P})$ case.

To be more precise about this risk measure, let $l: \mathbb{R} \rightarrow \mathbb{R}_{+}$be an increasing convex loss function, not identically constant. For some $x_{0} \in \mathbb{R}$, we define the acceptance set

$$
\mathcal{A}:=\left\{X \in L^{p}: \mathbb{E}[l(-X)] \leq l\left(x_{0}\right)\right\},
$$

and the corresponding shortfall convex risk measure $\rho: L^{p}(\mathbb{P}) \rightarrow \mathbb{R} \cup\{+\infty\}$

$$
\rho(X):=\inf \{\xi \in \mathbb{R}: X+\xi \in \mathcal{A}\}=\inf \left\{\xi \in \mathbb{R}: \mathbb{E}[l(-X-\xi)] \leq l\left(x_{0}\right)\right\},
$$

with the convention inf $\varnothing=+\infty$. Actually, we will work under the following assumption on $l$ :

$$
\lim _{x \rightarrow-\infty} l(x)=\underline{l} \leq l\left(x_{0}\right) \text { and } \quad \lim _{x \rightarrow+\infty} l(x)=+\infty .
$$

Note that Jensen's inequality implies that

$$
\left\{\xi \in \mathbb{R}: \mathbb{E}[l(-X-\xi)] \leq l\left(x_{0}\right)\right\} \subset\left\{\xi \in \mathbb{R}: l(-\mathbb{E}[X]-\xi) \leq l\left(x_{0}\right)\right\} .
$$

Moreover, we clearly have $\inf \left\{\xi \in \mathbb{R}: l(-\mathbb{E}[X]-\xi) \leq l\left(x_{0}\right)\right\}=-\mathbb{E}[X]-x_{0}$. Hence, the set $\left\{\xi \in \mathbb{R}: \mathbb{E}[l(-X-\xi)] \leq l\left(x_{0}\right)\right\}$ is bounded from below. Now, if $\mathbb{E}[l(-X)]<+\infty$, Lebesgue's dominated convergence theorem implies that

$$
\lim _{\xi \rightarrow+\infty} \mathbb{E}[l(-X-\xi)]=\underline{l} \leq l\left(x_{0}\right)
$$

so that, if $\underline{l}<l\left(x_{0}\right)$ then $\left\{\xi \in \mathbb{R}: \mathbb{E}[l(-X-\xi)] \leq l\left(x_{0}\right)\right\} \neq \varnothing$ hence $\rho(X) \in \mathbb{R}$, otherwise $\rho(X)=$ $+\infty$. Moreover, if we suppose that $\forall M>0, \mathbb{E}[l(-X+M)]<\infty$, then the function $\xi \mapsto$ $\mathbb{E}[l(-X-\xi)]$ is continuous and $\rho(X)$ is the lowest solution of the equation:

$$
\mathbb{E}[l(-X-\xi)]=l\left(x_{0}\right) .
$$


Classical examples are the entropic risk measure defined by setting $l(x)=e^{\lambda x}, x \in \mathbb{R}, \lambda>0$, $x_{0} \in \mathbb{R}$, the solution of (3) is unique and given by:

$$
\rho(X)=\frac{1}{\lambda} \log \mathbb{E}\left[e^{-\lambda\left(X+x_{0}\right)}\right],
$$

and the $l^{p}$-shortfall risk measure, defined by setting $l(x)=x^{p} \mathbf{1}_{\{x \geq 0\}}, x \in \mathbb{R}, p \geq 1, x_{0} \geq 0$.

Given an initial wealth $v \in \mathbb{R}$ and a trading strategy $\theta$, the resulting loss induces by $H$ is just the difference between the value of the contingent claim and the value of the portfolio at maturity $N: H-V_{N}^{v, \theta}$. The basic problem for the holder of the portfolio is to find an optimal (self-financed) strategy $\theta^{*}$ which minimizes the risk of the residual loss, i.e. a solution to the following minimization problem

$$
\inf _{\theta \in \mathcal{A}_{\mathcal{F}}} \rho\left(H-V_{N}^{v, \theta}\right)=\inf _{\theta \in \mathcal{A}_{\mathcal{F}}} \inf \left\{\xi \in \mathbb{R}: \mathbb{E}\left[l\left(-H+V_{N}^{v, \theta}-\xi\right)\right] \leq l\left(x_{0}\right)\right\}
$$

where $\mathcal{A}_{\mathcal{F}}=\left\{\theta=\left(\theta_{t}\right)_{1 \leq t \leq N}: \theta_{t} \in \Theta_{t-1}, t=1, \cdots, N\right\}$ is the set of admissible strategies and $\rho$ is a shortfall risk measure defined by (10). Let us note that the cash invariance property of $\rho$ implies

that for any initial wealth $v \in \mathbb{R}, \rho\left(H-V_{N}^{v, \theta}\right)=\rho\left(H-V_{N}^{0, \theta}\right)+v$. Hence, a solution of (15) (if any exists) does not depend on $v$. Consequently, without loss of generality, we will assume that the initial wealth $v$ is equal to 0 and we will write $V_{N}^{\theta}$ for $V_{N}^{0, \theta}$ throughout the rest of the paper.

Remark 2.1. Most of the cited papers consider the case of non-negative contingent claims and, more generally, impose admissibility condition on the value of the portfolio. Here, though in a discrete-time context, we consider a more general framework since we allow for contingent claims with a non-constant sign and we do not introduce admissibility condition on the portfolio so that the static risk minimization problem (5) can not be solved using Neyman-Pearson lemma.

We will impose the following absence of arbitrage property (NA):

$$
(\mathrm{NA}): \forall \theta \in \mathcal{A}_{\mathcal{F}}, \quad\left(V_{N}^{0, \theta} \geq 0 \text { a.s. } \Longrightarrow V_{N}^{0, \theta}=0 \text { a.s. }\right)
$$

There is a well-known duality result between the no-arbitrage property (6) and the existence of some equivalent martingale measure, it is the so-called fundamental theorem of asset pricing. It was proved in discrete time financial market models by several authors, see e.g. [42, [40] and [24] among others. We will denote by $\mathcal{P}(\mathbb{P})$ the class of all probability measures on $(\Omega, \mathcal{F})$ which are equivalent to $\mathbb{P}$ and under which the (discounted) price process $S$ is a martingale. Hence, (NA) implies that $\mathcal{P}(\mathbb{P}) \neq \varnothing$.

\subsection{A two steps control stochastic problem}

Looking at the right hand side of (5), a natural idea in order to solve the shortfall risk minimization problem (5) is to proceed in two steps as follows:

- the first step consists in solving for every $\xi \in \mathbb{R}$, the following stochastic control problem,

$$
\inf _{\theta \in \mathcal{A}_{\mathcal{F}}} \mathbb{E}\left[l\left(-H+V_{N}^{\theta}-\xi\right)\right]
$$

- once computed an optimal solution to (7) (if any), say $\theta^{*}(\xi), \xi \in \mathbb{R}$, the second step consists in finding the lowest solution $\xi^{*}$ of the following equation

$$
\inf _{\theta \in \mathcal{A}_{\mathcal{F}}} \mathbb{E}\left[l\left(-H+V_{N}^{\theta}-\xi\right)\right]=\mathbb{E}\left[l\left(-H+V_{N}^{\theta^{*}(\xi)}-\xi\right)\right]=l\left(x_{0}\right) .
$$


Next proposition shows that, as expected, proceeding following these two steps provides an optimal strategy to the risk minimization problem (5).

Proposition 2.1. Let $l: \mathbb{R} \rightarrow \mathbb{R}_{+}$be a non-decreasing convex function satisfying (2). Suppose that (NA) is satisfied. Assume that $\forall \xi \in \mathbb{R}, \exists \theta^{*}(\xi) \in \mathcal{A}_{\mathcal{F}}$ such that $\mathbb{E}\left[l\left(-H+V_{N}^{\theta^{*}(\xi)}-\xi\right)\right]=$ $\inf _{\theta \in \mathcal{A}_{\mathcal{F}}} \mathbb{E}\left[l\left(-H+V_{N}^{\theta}-\xi\right)\right]<+\infty$. Then, we have

$$
\inf _{\theta \in \mathcal{A}_{\mathcal{F}}} \rho\left(H-V_{N}^{\theta}\right)=\inf \left\{\xi \in \mathbb{R}: \inf _{\theta \in \mathcal{A}_{\mathcal{F}}} \mathbb{E}\left[l\left(-H+V_{N}^{\theta}-\xi\right)\right] \leq l\left(x_{0}\right)\right\}=\xi^{*}
$$

where $\xi^{*}$ is the lowest solution of (8).

Proof. First note that for every $\xi \in \mathbb{R}, \theta \in \mathcal{A}_{\mathcal{F}}$,

$$
\mathbb{E}\left[l\left(-H+V_{N}^{\theta^{*}(\xi)}-\xi\right)\right]=\inf _{\theta \in \mathcal{A}_{\mathcal{F}}} \mathbb{E}\left[l\left(-H+V_{N}^{\theta}-\xi\right)\right] \leq \mathbb{E}\left[l\left(-H+V_{N}^{\theta}-\xi\right)\right],
$$

so that,

$$
\left\{\xi \in \mathbb{R}: \mathbb{E}\left[l\left(-H+V_{N}^{\theta}-\xi\right)\right] \leq l\left(x_{0}\right)\right\} \subset\left\{\xi \in \mathbb{R}: \inf _{\theta \in \mathcal{A}_{\mathcal{F}}} \mathbb{E}\left[l\left(-H+V_{N}^{\theta}-\xi\right)\right] \leq l\left(x_{0}\right)\right\}
$$

Now, let $\mathbb{P}^{*} \in \mathcal{P}(\mathbb{P})$ such that $\frac{d \mathbb{P}^{*}}{d \mathbb{P}^{2}}<K$ a.s. for some deterministic constant $K<+\infty$. The existence of such probability measure is established in [24], Theorem 3 p.264. Consequently, using Jensen's inequality one finds that

$$
\mathbb{E}\left[l\left(-H+V_{N}^{\theta^{*}(\xi)}-\xi\right)\right]=\mathbb{E}_{\mathbb{P}^{*}}\left[l\left(-H+V_{N}^{\theta^{*}(\xi)}-\xi\right) \frac{d \mathbb{P}^{\mathbb{P}}}{d \mathbb{P}^{*}}\right]>\frac{1}{K} l\left(-\mathbb{E}_{\mathbb{P}^{*}}[H]-\xi\right),
$$

and since $\mathbb{E}_{\mathbb{P}^{*}}[|H|]<K \mathbb{E}[|H|]<+\infty$, we obtain that $\lim _{\xi \rightarrow-\infty} \inf _{\theta \in \mathcal{A}_{\mathcal{F}}} \mathbb{E}\left[l\left(-H+V_{N}^{\theta}-\xi\right)\right]=+\infty$, which finally implies that the set $\left\{\xi \in \mathbb{R}: \inf _{\theta \in \mathcal{A}_{\mathcal{F}}} \mathbb{E}\left[l\left(-H+V_{N}^{\theta}-\xi\right)\right] \leq l\left(x_{0}\right)\right\}$ is bounded from below. For $\xi \geq 0$, we have $l\left(-H+V_{N}^{\theta^{*}(0)}-\xi\right) \leq l\left(-H+V_{N}^{\theta^{*}(0)}\right) \in L^{1}(\mathbb{P})$, so that Lebesgue's dominated convergence theorem implies that

$$
\lim _{\xi \rightarrow+\infty} \inf _{\theta \in \mathcal{A}_{\mathcal{F}}} \mathbb{E}\left[l\left(-H+V_{N}^{\theta}-\xi\right)\right] \leq \lim _{\xi \rightarrow+\infty} \mathbb{E}\left[l\left(-H+V_{N}^{\theta^{*}(0)}-\xi\right)\right]=\underline{l} \leq l\left(x_{0}\right) .
$$

Hence, we have $\inf \left\{\xi \in \mathbb{R}: \inf _{\theta \in \mathcal{A}_{\mathcal{F}}} \mathbb{E}\left[l\left(-H+V_{N}^{\theta}-\xi\right)\right] \leq l\left(x_{0}\right)\right\} \in \mathbb{R} \cup\{+\infty\}$. Consequently, for all $\theta \in \mathcal{A}_{\mathcal{F}}$, we always have

$$
\rho\left(H-V_{N}^{\theta}\right) \geq \inf \left\{\xi \in \mathbb{R}: \inf _{\theta \in \mathcal{A}_{\mathcal{F}}} \mathbb{E}\left[l\left(-H+V_{N}^{\theta}-\xi\right)\right] \leq l\left(x_{0}\right)\right\},
$$

which implies that: $\inf _{\theta \in \mathcal{A}_{\mathcal{F}}} \rho\left(H-V_{N}^{\theta}\right) \geq \inf \left\{\xi \in \mathbb{R}: \inf _{\theta \in \mathcal{A}_{\mathcal{F}}} \mathbb{E}\left[l\left(-H+V_{N}^{\theta}-\xi\right)\right] \leq l\left(x_{0}\right)\right\}$. Now, the function $\xi \mapsto \inf _{\theta \in \mathcal{A}_{\mathcal{F}}} \mathbb{E}\left[l\left(-H+V_{N}^{\theta}-\xi\right)\right]$ is non-increasing, convex and finite-valued, hence, it is continuous. Consequently, the infimum in the right-hand side of (9) is reached at some point $\xi^{*}$, which is the lowest solution of the equation

$$
\inf _{\theta \in \mathcal{A}_{\mathcal{F}}} \mathbb{E}\left[l\left(-H+V_{N}^{\theta}-\xi\right)\right]=l\left(x_{0}\right) .
$$

It is easy to see that the strategy $\theta^{*}\left(\xi^{*}\right) \in \mathcal{A}_{\mathcal{F}}$ satisfies the following inequality

$$
\xi^{*}:=\inf \left\{\xi: \inf _{\theta \in \mathcal{A}_{\mathcal{F}}} \mathbb{E}\left[l\left(-H+V_{N}^{\theta}-\xi\right)\right] \leq l\left(x_{0}\right)\right\} \leq \inf \left\{\xi: \mathbb{E}\left[l\left(-H+V_{N}^{\theta^{*}\left(\xi^{*}\right)}-\xi\right)\right] \leq l\left(x_{0}\right)\right\},
$$


and it is clear that $\xi^{*} \in\left\{\xi: \mathbb{E}\left[l\left(-H+V_{N}^{\theta^{*}\left(\xi^{*}\right)}-\xi\right)\right] \leq l\left(x_{0}\right)\right\}$, which implies

$$
\xi^{*}=\inf \left\{\xi: \mathbb{E}\left[l\left(-H+V_{N}^{\theta^{*}\left(\xi^{*}\right)}-\xi\right)\right] \leq l\left(x_{0}\right)\right\}=\rho\left(H-V_{N}^{\theta^{*}\left(\xi^{*}\right)}\right) .
$$

Conversely, the strategy $\theta^{*}\left(\xi^{*}\right) \in \mathcal{A}_{\mathcal{F}}$ always satisfies: $\inf _{\theta \in \mathcal{A}_{\mathcal{F}}} \rho\left(H-V_{N}^{\theta}\right) \leq \rho\left(H-V_{N}^{\theta^{*}\left(\xi^{*}\right)}\right)=\xi^{*}$ which yields the announced equality: $\inf _{\theta \in \mathcal{A}_{\mathcal{F}}} \rho\left(H-V_{N}^{\theta}\right)=\rho\left(H-V_{N}^{\theta^{*}\left(\xi^{*}\right)}\right)=\xi^{*}$.

\subsection{Minimizing expected loss: a dynamic programming approach}

In this section, we prove the existence of an optimal strategy for the stochastic control problem (7) using an argument based on a dynamic programming principle in a non Markovian framework (see [16], [37]). The only result which is not elementary and that will be used is a measurable selection theorem.

Assumption 2.1. For every $\xi \in \mathbb{R}, \exists \bar{\theta}(\xi) \in \mathcal{A}_{\mathcal{F}}$ such that $\mathbb{E}\left[l\left(-H+V_{N}^{\bar{\theta}(\xi)}-\xi\right)\right]<+\infty$.

Working with regular conditional expectations, we will denote by $D_{t}(w)$ the smallest affine subspace of $\mathbb{R}^{d}$ containing the support of the regular conditional distribution of $\Delta S_{t}$ with respect to $\mathcal{F}_{t-1}$. Under (NA), one shows that it is an $\mathcal{F}_{t-1}$-measurable random vector subspace of $\mathbb{R}^{d}$ (see 24] for more details). We will assume throughout the rest of the paper that $D_{t} \neq\{0\}$, a.s., for $t=1, \cdots, N$.

In order to prove the existence of an optimal strategy $\theta^{*}(\xi), \xi \in \mathbb{R}$, that minimizes the expected loss, we introduce the following Bellman's function:

$$
\varphi_{N}\left(\xi, \theta_{1}, \cdots, \theta_{N}\right):=l\left(-H+V_{N}^{\theta}-\xi\right), \text { a.s. }
$$

for all $\xi \in \mathbb{R}, \theta \in \mathcal{A}_{\mathcal{F}}$; for $0 \leq t<N$ and for all $\xi \in \mathbb{R},\left(\theta_{1}, \cdots, \theta_{t}\right) \in \Theta_{0} \times \cdots \times \Theta_{t-1}$,

$$
\varphi_{t}\left(\xi, \theta_{1}, \cdots, \theta_{t}\right):=\operatorname{essinf}_{\theta_{t+1} \in \Theta_{t}} \mathbb{E}\left[\varphi_{t+1}\left(\xi, \theta_{1}, \cdots, \theta_{t+1}\right) \mid \mathcal{F}_{t}\right], \quad \text { a.s. }
$$

We will need the following characterization of (NA) whose statement and proof may be found in [37, see Proposition 3.3; see also [24]. We recall it here for sake of completeness.

Lemma 2.2. Suppose that $(N A)$ is satisfied. Then, for $t=0, \cdots, N-1$, there exists $\mathcal{F}_{t}$-measurable random variables $\beta_{t}, \kappa_{t}>0$ such that

$$
\forall u_{t+1} \in \Theta_{t} \cap D_{t+1}, \text { such that }\left|u_{t+1}\right|=1 \text { a.s., } \quad \mathbb{P}\left(u_{t+1} . \Delta S_{t+1}>\beta_{t} \mid \mathcal{F}_{t}\right) \geq \kappa_{t} \quad \text { a.s. }
$$

We will also need a measurable selection theorem, see e.g. [9] Theorem 82, p. 252, for a first version. Actually, we will use a slightly different version that is stated in [24] (see p.266 Lemma 5) which is more suited for our purposes.

Lemma 2.3. Let $G$ be a polish space (i.e. a complete separable metric space) with its Borel $\sigma$-field $\mathcal{G}$. Let $(H, \mathcal{H}, \mu)$ be a measure space and let $A$ be an $\mathcal{H} \otimes \mathcal{G}$-measurable subset of $H \times G$, with $H$-projection $\pi(A)=\{x: \exists y,(x, y) \in A\}$. There exists an $G$-valued $\mathcal{H}$-measurable function $Y$ such that $(x, Y(x)) \in A$ for $\mu$-almost all $x \in \pi(A)$.

Proposition 2.4. Let $l: \mathbb{R} \rightarrow \mathbb{R}_{+}$be a non-decreasing convex function satisfying (2). Suppose that (NA), Assumption 2.1 are satisfied and that the conditional distribution of $\Delta S_{t+1}$ given $\mathcal{F}_{t}$ is continuous (no affine hyperplane has positive mass) for $0 \leq t<N$. The functions

$$
\left(\theta_{t+1}, \cdots, \theta_{N}\right) \mapsto \mathbb{E}\left[l\left(-H+\sum_{\ell=t+1}^{N} \theta_{\ell} \cdot \Delta S_{\ell}+X-\xi\right) \mid \mathcal{F}_{t}\right], \quad 0 \leq t<N,
$$


are lower semi-continuous (l.s.c.), convex and satisfy

$$
\lim _{\substack{\left|\left(\theta_{t+1}, \cdots, \theta_{N}\right)\right| \rightarrow+\infty \\\left(\theta_{t+1}, \cdots, \theta_{N}\right) \in \Theta_{t} \times \cdots \times \Theta_{N-1}}} \mathbb{E}\left[l\left(-H+\sum_{\ell=t+1}^{N} \theta_{\ell} \cdot \Delta S_{\ell}+X-\xi\right) \mid \mathcal{F}_{t}\right]=+\infty, \quad \text { a.s. }
$$

for any $\xi \in \mathbb{R}$ and any $\mathcal{F}_{t}$-measurable $\mathbb{R}$-valued random variable $X$.

Proof. The convexity follows easily from the convexity of $l$. Let $t \in\{0, \cdots, N-1\}$ and $\xi \in \mathbb{R}$. The lower semi-continuity of this function is equivalent to its sequential lower semi-continuity. Take a sequence $\left(\theta_{t+1}^{k}, \cdots, \theta_{N}^{k}\right)_{k \geq 0}$ of $\Theta_{t} \times \cdots \times \Theta_{N-1}$ such that $\left(\theta_{t+1}^{k}, \cdots, \theta_{N}^{k}\right) \rightarrow\left(\theta_{t+1}, \cdots, \theta_{N}\right)$. The continuity of $l$ and conditional Fatou's Lemma implies that

$$
\liminf _{k} \mathbb{E}\left[l\left(-H+\sum_{\ell=t+1}^{N} \theta_{\ell}^{k} \cdot \Delta S_{\ell}+X-\xi\right) \mid \mathcal{F}_{t}\right] \geq \mathbb{E}\left[l\left(-H+\sum_{\ell=t+1}^{N} \theta_{\ell} \cdot \Delta S_{\ell}+X-\xi\right) \mid \mathcal{F}_{t}\right] \text { a.s. }
$$

Hence, $\left(\theta_{t+1}, \cdots, \theta_{N}\right) \mapsto \mathbb{E}\left[l\left(-H+\sum_{\ell=t+1}^{N} \theta_{\ell} \cdot \Delta S_{\ell}+X-\xi\right) \mid \mathcal{F}_{t}\right](w)$ is 1.s.c, for $\mathbb{P}$-almost all $w$.

For the last statement, following the lines of the proof of Proposition 2.1, we consider $\mathbb{P}^{*} \in \mathcal{P}(\mathbb{P})$ such that $\frac{d \mathbb{P}^{*}}{d \mathbb{P}^{\mathbb{P}}}<K$ a.s. for some $K<+\infty$. One may assume that there exists $I \subseteq\{t+1, \cdots, N\}$ such that $\left|\theta_{i}\right| \rightarrow+\infty$, for all $i \in I$. Denote by $\underline{i}=\min I \geq t+1$. Using Baye's rule and conditional Jensen's inequality, we have

$$
\begin{aligned}
& \mathbb{E}\left[l\left(-H+\sum_{\ell=t+1}^{N} \theta_{\ell} \cdot \Delta S_{\ell}+X-\xi\right) \mid \mathcal{F}_{\underline{i}}\right]=\mathbb{E}_{\mathbb{P}^{*}}\left[l\left(-H+\sum_{\ell=t+1}^{N} \theta_{\ell} \cdot \Delta S_{\ell}+X-\xi\right) \frac{d \mathbb{P}}{d \mathbb{P}^{*}} \mid \mathcal{F}_{\underline{i}}\right] \\
& \times \mathbb{E}\left[\frac{d \mathbb{P}^{*}}{d \mathbb{P}} \mid \mathcal{F}_{\underline{i}}\right] \\
& \geq \frac{1}{K} l\left(-\mathbb{E}_{\mathbb{P} *}\left[H \mid \mathcal{F}_{\underline{i}}\right]+\sum_{\ell=t+1}^{\underline{i}} \theta_{\ell} \cdot \Delta S_{\ell}+X-\xi\right) \\
& \times \mathbb{E}\left[\frac{d \mathbb{P}^{*}}{d \mathbb{P}} \mid \mathcal{F}_{\underline{i}}\right],
\end{aligned}
$$

which leads to the following inequality,

$$
\begin{array}{r}
\mathbb{E}\left[l\left(-H+\sum_{\ell=t+1}^{N} \theta_{\ell} \cdot \Delta S_{\ell}+X-\xi\right) \mid \mathcal{F}_{\underline{i}-1}\right] \geq \frac{1}{K} \mathbb{E}\left[l\left(-\mathbb{E}_{\mathbb{P}^{*}}\left[H \mid \mathcal{F}_{\underline{i}}\right]+\sum_{\ell=t+1}^{\underline{i}} \theta_{\ell} \cdot \Delta S_{\ell}+X-\xi\right)\right. \\
\left.\times \frac{d \mathbb{P}^{*}}{d \mathbb{P}} \mid \mathcal{F}_{\underline{\underline{i}}-1}\right] .
\end{array}
$$

Now, the convexity of $l$ implies that

$2 l\left(\frac{1}{2} \theta_{\underline{i}} . \Delta S_{\underline{i}}\right) \leq l\left(-\mathbb{E}_{\mathbb{P} *}\left[H \mid \mathcal{F}_{\underline{i}}\right]+\sum_{\ell=t+1}^{\underline{i}} \theta_{\ell} \cdot \Delta S_{\ell}+X-\xi\right)+l\left(\mathbb{E}_{\mathbb{P} *}\left[H \mid \mathcal{F}_{\underline{i}}\right]-\sum_{\ell=t+1}^{\underline{i}-1} \theta_{\ell} \cdot \Delta S_{\ell}-X+\xi\right)$,

so that it is sufficient to prove that

$$
\lim _{\left|\theta_{\underline{\theta}}\right| \rightarrow+\infty} \mathbb{E}\left[l\left(\frac{1}{2} \theta_{\underline{i}} . \Delta S_{\underline{i}}\right) \frac{d \mathbb{P}^{*}}{d \mathbb{P}} \mid \mathcal{F}_{\underline{i}-1}\right]=+\infty, \text { a.s. }
$$


Suppose that $\left|\theta_{\underline{i}}\right| \neq 0$, a.s., since $l$ is a non-decreasing and non-negative function, we have

$$
\left.\left.l\left(\frac{1}{2} \theta_{\underline{i}} \cdot \Delta S_{\underline{i}}\right) \geq l\left(\frac{1}{2}\left|\theta_{\underline{\underline{i}}}\right| \frac{\theta_{\underline{i}}}{\left|\theta_{\underline{i}}\right|} \cdot \Delta S_{\underline{i}}\right) \mathbf{1}_{\left\{\frac{\theta_{\underline{i}}}{\left|\theta_{\underline{\underline{i}}}\right|} \cdot \Delta S_{\underline{i}}>\beta_{\underline{i}}-1\right.}\right\} \geq l\left(\frac{1}{2}\left|\theta_{\underline{\underline{i}}}\right| \beta_{\underline{i}-1}\right) \mathbf{1}_{\left\{\frac{\theta_{\underline{i}}}{\mid \theta_{\underline{\underline{i}}}} \cdot \Delta S_{\underline{i}}>\beta_{\underline{i}}-1\right.}\right\},
$$

which leads to

$$
\begin{aligned}
\mathbb{E}\left[l\left(\frac{1}{2} \theta_{\underline{i}} . \Delta S_{\underline{i}}\right) \frac{d \mathbb{P}^{*}}{d \mathbb{P}} \mid \mathcal{F}_{\underline{i}-1}\right] & \geq l\left(\frac{1}{2}\left|\theta_{\underline{i}}\right| \beta_{\underline{i}-1}\right) \mathbb{P}^{*}\left(\frac{\theta_{\underline{i}}}{\mid \theta_{\underline{i}}}\left|\Delta S_{\underline{i}}>\beta_{\underline{i}-1}\right| \mathcal{F}_{\underline{i}-1}\right) \mathbb{E}\left[\frac{d \mathbb{P}^{*}}{d \mathbb{P}} \mid \mathcal{F}_{\underline{i}-1}\right] \\
& \geq l\left(\frac{1}{2}\left|\theta_{\underline{i}}\right| \beta_{\underline{i}-1}\right) \underset{\substack{u_{\underline{i}} \in \Theta_{\underline{i}} \cap D_{\underline{i}},\left|u_{\underline{i}}\right|=1}}{\operatorname{essinf}} \mathbb{P}^{*}\left(u_{\underline{i}} . \Delta S_{\underline{i}}>\beta_{\underline{i}-1} \mid \mathcal{F}_{\underline{i}-1}\right) \mathbb{E}\left[\frac{d \mathbb{P}^{*}}{d \mathbb{P}} \mid \mathcal{F}_{\underline{i}-1}\right] .
\end{aligned}
$$

Since the conditional distribution of $\Delta S_{\underline{\underline{i}}}$ given $\mathcal{F}_{\underline{i}-1}$ is continuous, $u \mapsto \mathbb{P}^{*}\left(u . \Delta S_{\underline{i}}>\beta_{\underline{i}-1} \mid \mathcal{F}_{\underline{i}-1}\right)(w)$ is continuous on the compact set $\mathcal{S}_{d}(0,1) \cap D_{\underline{i}}(w)$ for $\mathbb{P}$-almost all $w$, where $\mathcal{S}_{d}(0,1):=\left\{u \in \mathbb{R}^{d}:|u|=1\right\}$ denotes the (compact) unit sphere. Hence, there exists some $\bar{u}_{\underline{i}} \in \Theta_{\underline{i}} \cap D_{\underline{i}}$ such that, $\left|\bar{u}_{\underline{i}}\right|=1$, a.s. and

$$
\mathbb{P}^{*}\left(\bar{u}_{\underline{i}} . \Delta S_{\underline{i}}>\beta_{\underline{i}-1} \mid \mathcal{F}_{\underline{i}-1}\right)=\underset{u_{\underline{i}} \in \Theta_{\underline{i}}^{i} \cap D_{\underline{i}},\left|u_{\underline{i}}\right|=1}{\operatorname{essinf}} \mathbb{P}^{*}\left(u_{\underline{i}} . \Delta S_{\underline{i}}>\beta_{\underline{i}-1} \mid \mathcal{F}_{\underline{i}-1}\right) \quad \text { a.s. }
$$

Now, using Lemma 2.2 , we clearly get $\mathbb{P}\left(\bar{u}_{\underline{i}} . \Delta S_{\underline{i}}>\beta_{\underline{i}-1} \mid \mathcal{F}_{\underline{i}-1}\right)>0$, a.s., consequently we have $\mathbb{P}^{*}\left(\bar{u}_{\underline{i}} . \Delta S_{\underline{i}}>\beta_{\underline{i}-1} \mid \mathcal{F}_{\underline{i}-1}\right)>0$, a.s. Hence, finally, we get that

$$
\lim _{\left|\theta_{\underline{i}}\right| \rightarrow+\infty} \mathbb{E}\left[l\left(\frac{1}{2} \theta_{\underline{i}} . \Delta S_{\underline{i}}\right) \frac{d \mathbb{P}^{*}}{d \mathbb{P}} \mid \mathcal{F}_{\underline{i}-1}\right]=+\infty, \text { a.s. }
$$

which implies,

$$
\lim _{\substack{\left|\theta_{i}\right| \rightarrow+\infty, \theta_{i} \in \Theta_{i-1}, i \in I}} \mathbb{E}\left[l\left(-H+\sum_{\ell=t+1}^{N} \theta_{\ell} \cdot \Delta S_{\ell}+X-\xi\right) \mid \mathcal{F}_{\underline{\underline{i}}-1}\right]=+\infty, \quad \text { a.s. }
$$

One concludes using the tower property of conditional expectation and conditional Fatou's Lemma.

Now we are in position to state the main result of this paper. We prove the existence of an optimal strategy to the stochastic control problem (17) (and consequently to the problem (5D) by means of the dynamic programming backward induction (10). The proof is postponed to appendix 5.1 and relies on classical arguments from stochastic control theory.

Theorem 2.5. Let $l: \mathbb{R} \rightarrow \mathbb{R}_{+}$be a non-decreasing convex function satisfying (2). Suppose that (NA), Assumption 2.1 are satisfied and that the conditional distribution of $\Delta S_{t+1}$ given $\mathcal{F}_{t}$ is continuous for $0 \leq t<N$. For all $0 \leq t<N, \xi \in \mathbb{R},\left(\theta_{1}, \cdots, \theta_{t}\right) \in \Theta_{0} \times \cdots \times \Theta_{t-1}$, there exists $\left(\theta_{t+1}^{*}, \cdots, \theta_{N}^{*}\right) \in \Theta_{t} \times \cdots \times \Theta_{N-1}$ such that

$$
\begin{aligned}
\varphi_{t}\left(\xi, \theta_{1}, \cdots, \theta_{t}\right) & =\mathbb{E}\left[l\left(-H+\sum_{\ell=t+1}^{N} \theta_{\ell}^{*} \cdot \Delta S_{\ell}-V_{t}^{\theta}-\xi\right) \mid \mathcal{F}_{t}\right] \\
& =\underset{\left(\theta_{t+1}, \cdots, \theta_{N}\right) \in \Theta_{t} \times \cdots \times \Theta_{N-1}}{\operatorname{essinf}} \mathbb{E}\left[l\left(-H+V_{N}^{\theta}-\xi\right) \mid \mathcal{F}_{t}\right],
\end{aligned}
$$

where $\theta_{t+1}^{*}(w)=\theta_{t+1}^{*}\left(\xi, V_{t}^{\theta}(w), w\right) \in \Theta_{t}$, for almost all $\xi \in \mathbb{R}$, and the $\mathbb{R}^{d}$-valued function $(\xi, x, w) \mapsto \theta_{t+1}^{*}(\xi, x, w)$ is $\mathcal{B}(\mathbb{R}) \otimes \mathcal{B}(\mathbb{R}) \otimes \mathcal{F}_{t}$-measurable. 
Let us note that since the function $\xi \mapsto \inf _{\theta \in \mathcal{A}_{\mathcal{F}}} \mathbb{E}\left[l\left(-H+V_{N}^{\theta}-\xi\right)\right]$ is non-increasing, convex and finite-valued by assumption 2.1, it is continuous. Hence, the infimum $\xi^{*} \in \mathbb{R} \cup\{+\infty\}$ of the set $\left\{\xi: \inf _{\theta \in \mathcal{A}_{\mathcal{F}}} \mathbb{E}\left[l\left(-H+V_{N}^{\theta}-\xi\right)\right] \leq l\left(x_{0}\right)\right\}$ is the lowest solution of

$$
\inf _{\theta \in \mathcal{A}_{\mathcal{F}}} \mathbb{E}\left[l\left(-H+V_{N}^{\theta}-\xi\right)\right]=l\left(x_{0}\right) .
$$

Now set $\phi_{1}^{*}:=\theta_{1}^{*}\left(\xi^{*}, v\right)$ and define inductively

$$
\phi_{t+1}^{*}:=\theta_{t+1}^{*}\left(\xi^{*}, V_{t}^{\phi^{*}}\right), \quad 1 \leq t \leq T-1 .
$$

The predictability of the process $\theta^{*}$ implies that the strategy $\phi^{*}$ is a $\mathcal{F}$-predictable process. Moreover, using a backward induction it is easy to see that

$$
\mathbb{E}\left[l\left(-H+V_{N}^{\phi^{*}}-\xi^{*}\right)\right]=\varphi_{0}\left(\xi^{*}\right)=\inf _{\theta \in \mathcal{A}} \mathbb{E}\left[l\left(-H+V_{N}^{\theta}-\xi^{*}\right)\right]=l\left(x_{0}\right) .
$$

Hence, $\phi^{*} \in \mathcal{A}$ is the optimal strategy for the risk minimization problem (5).

\subsection{First order conditions}

In this section, we want to find an analytical characterization of the optimal strategy $\theta^{*}$. We start by deriving the backward first order conditions associated to the backward dynamic programming equations (10). That will allow us to devise (later on) numerical procedures to estimate the quantities of interest in a Markovian framework.

In order to derive the backward dynamic programming equations associated to the first order conditions, we consider a family of regular conditional distributions $\left(\Pi_{\ell}\right)_{0 \leq \ell \leq N-1}$ where $\Pi_{\ell}(d y, d x):=$ $\Pi_{\ell}(w, d y, d x)$ denotes the regular conditional distribution of the $N+1$-tuple $\left(H, \Delta S_{1}, \cdots, \Delta S_{N}\right)$ given $\mathcal{F}_{\ell}$. We make the following assumption

Assumption 2.2. (i) The function l is continuously differentiable and satisfies: $\frac{l^{\prime}(x)}{l(x)} \leq C$, when $|x| \rightarrow+\infty$.

There exists $\alpha>1$ such that for every $0<t \leq N$ the two following properties are satisfied:

(ii) There exists $\left(\tilde{\theta}_{t+1}, \cdots, \tilde{\theta}_{N}\right) \in \Theta_{t} \times \cdots \times \Theta_{N-1}$ such that for every $R>0$,

$$
\sup _{\left(x, \theta_{t}\right) \in \bar{B}_{1}(0, R) \times \bar{B}_{d}(0, R)} \mathbb{E}\left[l^{2 \alpha}\left(-H+\sum_{\ell=t+1}^{N} \tilde{\theta}_{\ell} \cdot \Delta S_{\ell}+\theta_{t} \cdot \Delta S_{t}+x-\xi\right) \mid \mathcal{F}_{t-1}\right]<+\infty \text { a.s. }
$$

for any $\xi \in \mathbb{R}$, where $\bar{B}_{d}(0, R)$ denotes the closed ball of radius $R$ in $\mathbb{R}^{d}$,

(iii) and, $\mathbb{E}\left[\left|\Delta S_{t}\right|^{2 \alpha} \mid \mathcal{F}_{t-1}\right]<+\infty$ a.s.

Theorem 2.6. Suppose that the assumptions of Theorem 2.5 are satisfied as well as assumption 2.2 above. Then, for every $1 \leq t<N$, for every $\left(\theta_{1}, \cdots, \theta_{t-1}\right) \in \Theta_{0} \times \cdots \times \Theta_{t-2}$, for every $\xi \in \mathbb{R}$, the function $\theta_{t} \mapsto \mathbb{E}\left[\varphi_{t}\left(\theta_{1}, \cdots, \theta_{t}\right) \mid \mathcal{F}_{t-1}\right]$ is differentiable with a gradient given by

$$
\mathbb{E}\left[\Delta S_{t} l^{\prime}\left(-H+\sum_{\ell=t+1}^{N} \theta_{\ell}^{*} \cdot \Delta S_{\ell}+V_{t}^{\theta}-\xi\right) \mid \mathcal{F}_{t-1}\right] .
$$

Hence, the optimal strategy at time $t, \theta_{t}^{*} \in \Theta_{t}$ satisfies

$$
\mathbb{E}\left[\Delta S_{t} l^{\prime}\left(-H+\sum_{\ell=t+1}^{N} \theta_{\ell}^{*} \cdot \Delta S_{\ell}+\theta_{t}^{*} . \Delta S_{t}+V_{t-1}^{\theta}-\xi\right) \mid \mathcal{F}_{t-1}\right]=0 \text { a.s. }
$$

Once again the proof is postponed to appendix [5.2 and relies on classical arguments from stochastic control theory. 


\section{Computational and numerical aspects of shortfall risk hedging}

In this section, we are concerned by the problem of computing the shortfall risk and the optimal strategy for the risk minimization problem. In a first step, we propose a Robbins-Monro (RM) stochastic algorithm for estimating the shortfall risk. Then, we propose a method to estimate the optimal strategy and the shortfall risk for the special case of one-step trading strategies. Finally, we consider the case of dynamic strategies. We propose a (vector) quantization based dynamic programming algorithm which intensively relies at each time step on stochastic approximation or Newton-Raphson optimization algorithms.

\subsection{Estimating the shortfall risk: a Robbins-Monro algorithm}

In this part, we study the problem of computing the shortfall risk of a financial position $X \in L^{p}(\mathbb{P})$. We will assume that the function $l$ is strictly increasing and satisfies (2) with $\underline{l}<l\left(x_{0}\right)$ (otherwise $\rho(X)=+\infty$ for any $\left.X \in L^{p}(\mathbb{P})\right)$. Moreover, we suppose that for every $M>0, \mathbb{E}[l(-X+M)]<$ $+\infty$ so that the function $\xi \mapsto \mathbb{E}[l(-X-\xi)]$ is continuous. Hence, $\rho(X)$ is the unique solution of the equation:

$$
\mathbb{E}[l(-X-\xi)]=l\left(x_{0}\right) .
$$

We set $K(\xi, x):=l(-x-\xi)-l\left(x_{0}\right), x \in \mathbb{R}$. Since we are looking for $\xi$ for which $\mathbb{E}[K(\xi, X)]=0$, we may rely on the so-called Robbins-Monro algorithm to estimate such quantity. First, we need the following assumption

Assumption 3.1. There exists a non-negative continuous function $\varphi$ such that:

$$
\mathbb{E}\left[l^{2}(-X-\xi)\right] \leq C(1+\varphi(\xi)), \quad \xi \in \mathbb{R},
$$

for some constant $C>0$.

Then, we slightly modify the function $K$ by setting $H(\xi, x):=\frac{1}{(1+\varphi(\xi))^{1 / 2}} K(\xi, x), x \in \mathbb{R}$, so that we have

$$
\{\xi \in \mathbb{R}: \mathbb{E}[H(\xi, x)]=0\}=\{\xi \in \mathbb{R}: \mathbb{E}[K(\xi, x)]=0\} .
$$

Now, we are in position to implement the following RM algorithm to estimate $\rho(X)$ :

$$
\xi_{n}=\xi_{n-1}+\gamma_{n} H\left(\xi_{n-1}, X_{n}\right), \quad n \geq 1,
$$

where $\left(X_{n}\right)_{n \geq 1}$ is an i.i.d. sequence of random variables with the same distribution as $X$, independent of $\xi_{0}$, with $\mathbb{E}\left[\left|\xi_{0}\right|\right]<+\infty$ and $\left(\gamma_{n}\right)_{n \geq 1}$ is a positive deterministic sequence (decreasing to 0 ) satisfying

$$
\sum_{n \geq 1} \gamma_{n}=+\infty \text { and } \sum_{n \geq 1} \gamma_{n}^{2}<+\infty
$$

Remark 3.1. Assumption 3.1 is satisfied for a wide range of loss functions including the two classical examples $l(x)=e^{\lambda x}, \lambda>0\left(\right.$ take $\left.\varphi(\xi):=e^{-2 \lambda \xi}, \xi \in \mathbb{R}\right)$ and $l(x):=x^{p} \mathbf{1}_{x \geq 0}$ (take $\left.\varphi(\xi):=\xi^{2 p}, \xi \in \mathbb{R}\right)$, even if for the exponential case, $\rho(X)$ is explicitly known. This function $\varphi$ will play the role of a normalization factor with the well-known consequence in practice that if $\varphi$ is not a sharp upper bound and takes too large values compared to $l$, the algorithm may eventually freeze and the "CLT" regime will take place later than it would with a sharper upper bound.

Proposition 3.1. Suppose that Assumption 3.1 is fulfilled and that the step sequence $\left(\gamma_{n}\right)_{n \geq 1}$ satisfies (13). Then,

$$
\xi_{n} \stackrel{a . s .}{\longrightarrow} \rho(X), \quad n \rightarrow+\infty,
$$

where $\left(\xi_{n}\right)_{n \geq 0}$ is the recursive sequence defined by (12). 
Proof. We just have to check that the function $H$ of the algorithm (12) and its mean $h():.=$ $\mathbb{E}[H(., X)]$ satisfies the assumptions of the classical Robbins-Monro Theorem (see e.g. [13] or [29]):

- Mean reverting assumption: since the function $l$ is increasing for every $\xi \in \mathbb{R}, \xi \neq \rho(X)$, we have

$$
(\xi-\rho(X)) h(\xi)=\frac{1}{(1+\varphi(\xi))^{1 / 2}}(\xi-\rho(X))\left(\mathbb{E}[l(-X-\xi)]-l\left(x_{0}\right)\right)<0 .
$$

- Linear growth assumption: thanks to Assumption 3.1, the linear growth assumption of the function $\xi \mapsto\|H(., X)\|_{2}$ is fulfilled since this function is clearly bounded.

Consequently, Robbins-Monro's Theorem yields $\xi_{n} \stackrel{a . s}{\longrightarrow} \rho(X), n \rightarrow+\infty$ and in every $L^{p}, p \in$ $[1,2)$.

As concerns the rate of convergence, once again (12) is a regular stochastic approximation algorithm that behaves as described in usual Stochastic Approximation Theory textbooks, see e.g. [6], 12] or [29]. Hence, under standard assumptions, the sequence $\left(\xi_{n}\right)_{n \geq 0}$ satisfies a Central Limit Theorem (CLT) at rate $\sqrt{\gamma_{n}^{-1}}$. It suggests to set $\gamma_{n}=\frac{\alpha}{\beta+n}, n \geq 1, \alpha, \beta>0$. If the function $l$ satisfies $\mathbb{E}\left[l^{\prime}(-X+R)\right]<+\infty$, for every $R>0$, then one shows that $h$ is differentiable and

$$
h^{\prime}(\rho(X))=-\frac{1}{(1+\varphi(\rho(X)))^{1 / 2}} \mathbb{E}\left[l^{\prime}(-X-\rho(X))\right] .
$$

Now, general results about stochastic approximation algorithm say that if $\alpha>\frac{1}{-2 h^{\prime}(\rho(X))}$ then $\sqrt{n}\left(\xi_{n}-\rho(X)\right) \stackrel{\mathcal{L}}{\longrightarrow} \mathcal{N}\left(0, \sigma_{\alpha}^{2}\right)$ where $\sigma_{\alpha}^{2}=\operatorname{Var}(H(\rho(X), X)) \frac{\alpha^{2}}{-2 \alpha h^{\prime}(\rho(X))-1}$. The function $\alpha \mapsto \sigma_{\alpha}^{2}$ reaches its minimum for $\alpha^{*}=\frac{1}{-h^{\prime}(\rho(X))}$ leading to the optimal asymptotic variance

$$
\sigma_{*}^{2}=\sigma_{\alpha^{*}}^{2}=\frac{\operatorname{Var}(H(\rho(X), X))}{h^{\prime}(\rho(X))^{2}}=\frac{\mathbb{E}\left[\left(l(-X-\rho(X))-l\left(x_{0}\right)\right)^{2}\right]}{\mathbb{E}\left[l^{\prime}(-X-\rho(X))\right]^{2}}
$$

Hence, the optimal rate of convergence is not influenced by the normalizing function $\varphi$ but the choice of $\alpha$ is difficult since the quantity $h^{\prime}(\rho(X))$ is unknown to the user. This will induce a (more or less) blind choice for the constant $\alpha$. One solution to overcome this difficulty can be to use a rough estimate of the quantity $h^{\prime}(\rho(X))$ using very few iterations at the beginning of the algorithm. Actually, we didn't meet this difficulty in our numerical experiments.

A well-known method to circumvent the difficulties induced by the specification of $\alpha$, which are classical in this field, is to add one component to our first algorithm (12) by introducing the empirical mean of the sequence $\left(\xi_{n}\right)_{n \geq 1}$ implemented with a slowly decreasing step according to the averaging principle of Ruppert and Polyak (see e.g. [36], [41]). It consists in setting $\gamma_{n}=\frac{\alpha}{n^{c}}$, $\alpha>0, \frac{1}{2}<c<1$ and

$$
\bar{\xi}_{n+1}:=\frac{1}{n+1} \sum_{k=0}^{n} \xi_{k}=\bar{\xi}_{n}-\frac{1}{n+1}\left(\bar{\xi}_{n}-\xi_{n}\right), n \geq 0
$$

where $\left(\xi_{n}\right)_{n \geq 0}$ is the sequence defined by (12) starting at $\xi_{0}$. The Ruppert and Polyak's Averaging Principle says that the sequence $\left(\bar{\xi}_{n}\right)_{n \geq 0}$ (which converges toward $\rho(X)$ ) satisfies a CLT at the optimal rate $\sqrt{n}$ and with the optimal asymptotic variance (14). 


\subsection{Shortfall risk minimization using a one step strategy}

In this part, we are concerned by the computational aspects of the risk minimization problem in the special case where the holder of the portfolio faced with the contingent given by the $\mathcal{F}_{N}$-measurable random variable $H \in L^{p}(\mathbb{P})$ hedges his risk using a one step strategy decided at time $t=0$. For many reasons (transaction costs, difficulties to store energy assets, ...), the holder of a portfolio may not want to trade every day but may be only interested by a rough hedge to reduce its risk so it is an interesting case in practice. In energy markets, practitioners may be interested by a rough hedge of their loss using only few forward contracts (which are the only assets available for hedging purpose), especially when dealing with physical assets like gas storage or power plant. Moreover, this rough optimal portfolio will serve as a proxy to build (later on) dynamic hedging strategies.

- The general case: The problem (7) and (8) can be written

$$
\inf _{\theta \in \mathbb{R}^{d}} \mathbb{E}[l(-H+\theta \cdot X-\xi)]=l\left(x_{0}\right), \quad \text { with } X:=S_{N}-S_{0},
$$

which amounts, under the assumptions of Theorem 2.6, to the computation of $\left(\xi^{*}, \theta^{*}\left(\xi^{*}\right)\right) \in \mathbb{R} \times \mathbb{R}^{d}$ solution of the following systems of $d+1$ non-linear equations of $d+1$ unknowns

$$
\begin{aligned}
\mathbb{E}[l(-H+\theta \cdot X-\xi)] & =l\left(x_{0}\right) \\
\mathbb{E}\left[X l^{\prime}(-H+\theta \cdot X-\xi)\right] & =0
\end{aligned}
$$

For sake of simplicity, we assume that $l$ is increasing and strictly convex so that the above systems of non-linear equations admits an unique solution $\left(\xi^{*}, \theta^{*}\right)$ which will be the target of our algorithm.

We set $h(\xi, \theta):=\left(\mathbb{E}[l(-H+\theta \cdot X-\xi)]-l\left(x_{0}\right), \mathbb{E}\left[X l^{\prime}(-H+\theta \cdot X-\xi)\right]\right),(\xi, \theta) \in \mathbb{R} \times \mathbb{R}^{d}$. We assume that $l$ is twice differentiable and that for every $R>0$,

$$
\mathbb{E}\left[\left|X X^{T}\right| \sup _{\theta \in \bar{B}_{d}(0, R)} l^{\prime \prime}(-H+\theta \cdot X+R)\right]<+\infty, \quad \mathbb{E}\left[|X| l^{\prime}(-H+R|X|+R)\right]<+\infty .
$$

Under these assumptions, using Lebesgue's dominated convergence theorem, one shows that $h$ is differentiable and that $D h$ is given by

$$
D h(\xi, \theta)=\left(\begin{array}{cc}
-\mathbb{E}\left[l^{\prime}(-H+\theta \cdot X-\xi)\right] & \mathbb{E}\left[X l^{\prime}(-H+\theta \cdot X-\xi)\right] \\
-\mathbb{E}\left[X l^{\prime \prime}(-H+\theta \cdot X-\xi)\right] & \mathbb{E}\left[X X^{T} l^{\prime \prime}(-H+\theta \cdot X-\xi)\right]
\end{array}\right)
$$

One can approximate $h$ and $D h$ using Monte Carlo simulation with $n$ samples

$$
h_{n}(\xi, \theta)=\left(\frac{1}{n} \sum_{k=1}^{n} l\left(-H_{k}+\theta \cdot X_{k}-\xi\right)-l\left(x_{0}\right), \frac{1}{n} \sum_{k=1}^{n} X_{k} l^{\prime}\left(-H_{k}+\theta \cdot X_{k}-\xi\right)\right)
$$

and

$$
D h_{n}(\xi, \theta)=\left(\begin{array}{cc}
-\frac{1}{n} \sum_{k=1}^{n} l^{\prime}\left(-H_{k}+\theta \cdot X_{k}-\xi\right) & \frac{1}{n} \sum_{k=1}^{n} X_{k} l^{\prime}\left(-H_{k}+\theta \cdot X_{k}-\xi\right) \\
-\frac{1}{n} \sum_{k=1}^{n} X_{k} l^{\prime \prime}\left(-H_{k}+\theta \cdot X_{k}-\xi\right) & \frac{1}{n} \sum_{k=1}^{n} X_{k} X_{k}^{T} l^{\prime \prime}\left(-H_{k}+\theta \cdot X_{k}-\xi\right)
\end{array}\right) .
$$

where $\left(H_{k}, X_{k}\right)_{1 \leq k \leq n}$ is an i.i.d. sequence of $d+1$ random vectors having the distribution of $(H, X)$. If $\mathbb{P}(X \neq 0)>0$ and (17) is satisfied, then for $n$ large enough there is an index $k \in\{1, \cdots, n\}$ such that $X_{k} \neq 0$ so that the system of $d+1$ non-linear equations of $d+1$ unknowns $h_{n}(\xi, \theta)=0$ admits a unique solution $\left(\xi_{n}^{*}, \theta_{n}^{*}\right)$ and the matrix $D h_{n}\left(\xi_{n}^{*}, \theta_{n}^{*}\right)$ writes

$$
D h_{n}\left(\xi_{n}^{*}, \theta_{n}^{*}\right)=\left(\begin{array}{cc}
-\frac{1}{n} \sum_{k=1}^{n} l^{\prime}\left(-H_{k}+\theta_{n}^{*} \cdot X_{k}-\xi_{n}^{*}\right) & 0 \\
-\frac{1}{n} \sum_{k=1}^{n} X_{k} l^{\prime \prime}\left(-H_{k}+\theta_{n}^{*} \cdot X_{k}-\xi_{n}^{*}\right) & \frac{1}{n} \sum_{k=1}^{n} X_{k} X_{k}^{T} l^{\prime \prime}\left(-H_{k}+\theta_{n}^{*} \cdot X_{k}-\xi_{n}^{*}\right)
\end{array}\right) .
$$


and is invertible. As a consequence, by storing the random variables $\left(H_{k}, X_{k}\right)_{1 \leq k \leq n}$ in the computer memory, the solution $\left(\xi_{n}^{*}, \theta_{n}^{*}\right)$ can be computed with very good accuracy using few steps (usually 5 are enough) of Newton-Raphson's optimization algorithm. If $z_{0}:=\left(\xi_{0}, \theta_{0}\right)$ is sufficiently close to $\left(\xi_{n}^{*}, \theta_{n}^{*}\right)$ then if we set $z_{k}=\left(\xi_{k}, \theta_{k}\right)$ the recursive algorithm

$$
z_{k+1}=z_{k}-D h_{n}\left(z_{k}\right)^{-1} h_{n}\left(z_{k}\right), \quad z_{k}:=\left(\xi_{k}, \theta_{k}\right), \quad k \geq 0,
$$

is known to converge toward $\left(\xi_{n}^{*}, \theta_{n}^{*}\right)$.

Though this procedure is only locally convergent, Newton-Raphsons algorithm is attractive because it converges rapidly from any sufficiently good initial guess under standard assumptions. Our numerical results indicates that (21) converges quickly in few iterations and we didn't observe the failure of convergence in our examples. In order to obtain a globally convergent algorithm, one may devise the method of steepest descent as the Armijo rule as explained in classical Non-linear Optimization textbooks like [10], 31]. However, these methods requires more computational efforts than the classical Newton-Raphson's algorithm. Hence, in this paper, we only considered (21). This kind of Newton-Raphson's algorithm combined with Monte Carlo simulation has already been investigated in [25] as an alternative method to stochastic approximation algorithm to estimate the optimal change of measure in the context of variance reduction technique by importance sampling for normal random vectors. We will not enter into technicalities at this stage but it is possible to prove that $\left(\xi_{n}^{*}, \theta_{n}^{*}\right)$ a.s. converges toward $\left(\xi^{*}, \theta^{*}\right), n \rightarrow+\infty$, the unique solution of (15) and (16) following an easy adaptation of Proposition 1.2 in [25] which follows from the local uniform convergence of $h_{n}$ to the continuous function $h$ under the assumptions introduced above.

- The case of exponential loss function: Let us note that in the case of the exponential loss function $l(x):=e^{\lambda x}, \lambda>0, x \in \mathbb{R}$ the system (15), (16) can be greatly simplified:

$$
\xi^{*}=\frac{1}{\lambda} \log \left(\mathbb{E}\left[e^{\lambda\left(-H+\theta^{*} \cdot X-x_{0}\right)}\right]\right), \quad x_{0} \in \mathbb{R},
$$

where $\theta^{*}$ is the unique solution of $\mathbb{E}\left[X e^{\lambda(-H+\theta \cdot X)}\right]=0$. This optimal one step strategy can be estimated using a classical stochastic gradient algorithm derived from the Lyapunov function $L: \theta \mapsto \mathbb{E}\left[e^{\lambda(-H+\theta \cdot X)}\right]$. We suppose that $\mathbb{E}\left[|X|^{2} e^{-2 \lambda H+\lambda|X|^{2}}\right]<+\infty$, so that by setting

$$
K_{1}(\theta, h, x):=\phi(\theta) x e^{\lambda(-h+\theta \cdot x)}, \quad \text { with } \phi(\theta):=\frac{1}{\left(1+e^{2 \lambda|\theta|^{2}}\right)^{1 / 2}}
$$

we have $\left\{\theta: \mathbb{E}\left[K_{1}(\theta, H, X)\right]=0\right\}=\left\{\theta: \mathbb{E}\left[e^{\lambda(-H+\theta \cdot X)}\right]=0\right\}$ and one may devise the following recursive scheme

$$
\theta_{n+1}=\theta_{n}-\gamma_{n+1} K_{1}\left(\theta_{n}, H_{n+1}, X_{n+1}\right), \quad n \geq 0,
$$

where $\left(H_{n}, X_{n}\right)_{n \geq 1}$ is an i.i.d. sequence of random vectors with the same distribution as $(H, X)$, independent of $\theta_{0}$, with $\mathbb{E}\left[\left|\theta_{0}\right|\right]<+\infty$ and $\left(\gamma_{n}\right)_{n \geq 1}$ is a positive deterministic sequence (decreasing to 0) satisfying (13). A straightforward application of Robbins-Monro's theorem yields the a.s. convergence of the sequence $\left(\theta_{n}\right)_{n \geq 0}$ toward $\theta^{*}$ as $n \rightarrow+\infty$. A natural idea in order to estimate $\xi^{*}$ given by (22) is to devise an averaging compagnon procedure derived from (23) namely, $\xi_{0}=0$ and for $n=1,2, \cdots$,

$\xi_{n+1}:=\frac{1}{\lambda} \log \left(C_{n+1}\right)$ with $C_{n+1}:=\frac{1}{n+1} \sum_{k=0}^{n} e^{\lambda\left(-H_{k+1}+\theta_{k} \cdot X_{k+1}\right)}=C_{n}-\frac{1}{n+1} K_{2}\left(\theta_{n}, C_{n}, H_{n+1}, X_{n+1}\right)$ 
where $K_{2}(\theta, C, h, x):=C-e^{\lambda(-h+\theta \cdot x)}$. One can also consider a slight modification of (24) which consists in using both procedure with the same step size $\left(\gamma_{n}\right)_{n \geq 1}$ satisfying (13), as proposed in [2] for the recursive estimation of the Value-at-Risk and the Conditional Value-at-Risk. This leads to the more consistent procedure which can be written as for $n \geq 0$

$$
\left\{\begin{array}{l}
\theta_{n+1}=\theta_{n}-\gamma_{n+1} K_{1}\left(\theta_{n}, H_{n+1}, X_{n+1}\right), \theta_{0} \in \mathbb{R}^{d} \\
C_{n+1}=C_{n}-\gamma_{n+1} K_{2}\left(\theta_{n}, C_{n}, H_{n+1}, X_{n+1}\right), C_{0}=0 \\
\xi_{n+1}=\frac{1}{\lambda} \log \left(C_{n+1}\right)
\end{array}\right.
$$

Using similar argumentation as those used in [2] for the convergence of the VaR-CVaR procedure, one shows that the above recursive procedure satisfies $\left(\theta_{n}, C_{n}\right) \stackrel{a . s}{\longrightarrow}\left(\theta^{*}, C^{*}\right)$ as $n \rightarrow+\infty$, where $C^{*}=e^{\lambda \xi^{*}}$ and that the sequence of interest $\left(\theta_{n}, \xi_{n}\right)_{n \geq 1}$ satisfies a CLT under standard assumptions. Hence, our numerical results for the exponential case will be built on the procedure (3.2) instead of (21).

\subsection{Dynamic shortfall risk minimization in a Markovian framework}

In practical example, the $\mathbb{R}$-valued random variable $H$ can be written as a function of a process $\left(S_{t}, Z_{t}\right)_{1 \leq t \leq N}$, i.e. $H=\phi(S, Z)$, where $S$ is the underlying prices process and $Z$ is a hidden $\mathbb{R}^{q}$ valued and $\mathcal{F}$-measurable process. Typically, in the electricity market, $Z$ can be considered as the temperature process or some consumption process and $H$ may modelize the loss suffered by an energy company due to an anormal annual electricity (or gas) consumption. In this kind of modelization, $Z$ is the source of incompleteness of the financial market. We suppose that $\mathcal{F}_{t}:=$ $\sigma\left(\left(S_{s}, Z_{s}\right), s \leq t\right), t=1, \cdots, N$.

For simplicity's and clarity's sake, we will only consider the case of European contingent claim, i.e. the case where $H$ does not depend of the whole trajectory of the process $(S, Z)$ but only of its value at maturity $N$ so that we can write $H=\phi\left(S_{N}, Z_{N}\right)$. In order to simplify the numerical computation of conditional expectations that appear in the first order dynamic programming conditions (11), we will suppose that the process $(S, Z)$ is a Markov chain, as it is the case if it is a stochastic differential equation discretized using the Euler-Maruyama scheme, for instance. More specifically, we will suppose that for every $t=1, \cdots, N$ there exists two measurable functions $G_{t}: \mathbb{R}^{d} \times \mathbb{R}^{q} \times \mathbb{R}^{r_{t}} \rightarrow \mathbb{R}^{d}, F_{t}: \mathbb{R}^{d} \times \mathbb{R}^{q} \times \mathbb{R}^{r_{t}} \rightarrow \mathbb{R}$ such that

$$
S_{t}-S_{t-1}=G_{t}\left(S_{t-1}, Z_{t-1}, U_{t}\right) \quad \text { and } \quad H=F_{t}\left(S_{t-1}, Z_{t-1}, U_{t}\right), \quad\left(S_{0}, Z_{0}\right) \in \mathbb{R}^{d} \times \mathbb{R}^{q}
$$

where $U_{t}$ is an $r_{t}$-dimensional random variable independent of $\mathcal{F}_{t-1}$. Under this markovian framework, we introduce the following functions:

$$
V_{t}\left(\theta, v, s_{t-1}, z_{t-1}\right):=\mathbb{E}\left[v_{t}\left(\theta, v, s_{t-1}, z_{t-1}\right)\right],
$$

where

$$
\begin{aligned}
v_{t}\left(\theta, v, s_{t-1}, z_{t-1}\right):=G_{t} & \left(s_{t-1}, z_{t-1}, U_{t}\right) \\
& \times l^{\prime}\left(-F_{t}\left(s_{t-1}, z_{t-1}, U_{t}\right)+\sum_{\ell=t+1}^{N} \theta_{\ell}^{*} \cdot \Delta S_{\ell}+\theta \cdot G_{t}\left(s_{t-1}, z_{t-1}, U_{t}\right)+v\right)
\end{aligned}
$$

with $\theta \in \mathbb{R}^{d}, v \in \mathbb{R}, s_{t-1} \in \mathbb{R}^{d}, z_{t-1} \in \mathbb{R}^{q}$. With these notations, the first order condition (11) at time $t \in\{1, \cdots, N\}$ can be written a.s.

$$
\mathbb{E}\left[\Delta S_{t} l^{\prime}\left(-H+\sum_{\ell=t+1}^{N} \theta_{\ell}^{*} \cdot \Delta S_{\ell}+\theta_{t}^{*} \cdot \Delta S_{t}+V_{t-1}^{\theta}-\xi\right) \mid \mathcal{F}_{t-1}\right]=V_{t}\left(\theta_{t}^{*}, V_{t-1}^{\theta}-\xi, S_{t-1}, Z_{t-1}\right)=0 .
$$


Consequently, in order to compute $\theta_{t}^{*}$, we need to solve the local optimization problem (25) for each $V_{t-1}^{\theta}-\xi$ and $\left(S_{t-1}(w), Z_{t-1}(w)\right), w \in \Omega$. This highlights the numerical complexity in solving (25). In practice, one must discretize the values taken by $V_{t-1}^{\theta}-\xi,\left(S_{t-1}, Z_{t-1}\right)$ and is led to make some approximations.

When the dimension of the random vector to be generated $\left(S_{t-1}, Z_{t-1}\right)$, which is equal to $d+q$, is large (greater than 10 in practice), one can only rely on Monte Carlo simulations and solves (25) using $n$ samples

$$
V_{t}\left(\theta_{t}^{*, k}, V_{t-1}^{\theta}-\xi, S_{t-1}^{k}, Z_{t-1}^{k}\right)=0, \quad k=1, \cdots, n
$$

where $\left(S_{t-1}^{k}, Z_{t-1}^{k}\right)_{1 \leq k \leq n}$ are i.i.d. random vectors having the distribution of $\left(S_{t-1}, Z_{t-1}\right)$. The problem amounts to solving $n$ systems of $d$ non-linear equations of $d$ unknowns $\theta_{t}^{*, k}$.

When the dimension $\left(S_{t-1}, Z_{t-1}\right)$ is small, one may use an integration cubature formula based for instance on a spatial discretization of $\left(S_{t-1}, Z_{t-1}\right)$. A commonly used method in such context is optimal vector quantization. Thus, we consider an (possibly non optimal) $M_{t}$-quantization $\left(\hat{S}_{t-1}, \hat{Z}_{t-1}\right)$ of the random vector $\left(S_{t-1}, Z_{t-1}\right)$, where for $t=1, \cdots, N, M_{t}$ is a positive integer, based on a quantization grid $\Gamma_{t}:=\left(\left(s_{t-1}^{1}, z_{t-1}^{1}\right), \cdots,\left(s_{t-1}^{M_{t}}, z_{t-1}^{M_{t}}\right)\right)$. Then, one solves $M_{t}$ systems of $d$ non-linear equations of $d$ unknowns

$$
V_{t}\left(\theta_{t}^{*, j}, V_{t-1}^{\theta}-\xi, s_{t-1}^{j}, z_{t-1}^{j}\right)=0, \quad j=1, \cdots, M_{t} .
$$

For more details about optimal vector quantization, including error bounds for cubature formulas, we refer to [34. This first form (26) is not satisfactory from an algorithmic point of view. Indeed, in order to devise a fully implementable numerical procedure one has to discretize the real-valued $\mathcal{F}_{t-1}$-measurable random variable $V_{t-1}^{\theta}-\xi$. This is clearly impossible in all generality since we do not have access to its law and we don't know the value of $\left(\theta_{\ell}\right)_{1 \leq \ell \leq t-1}$. Hence, we are naturally led to make an approximation which consists in supposing that $V_{t-1}^{\theta}-\xi$ takes values in a prespecified (sufficiently large) interval $\left[\underline{v}_{t-1}, \bar{v}_{t-1}\right]$. Then, we consider a discrete grid $\left(v_{t-1}^{i}\right)_{1 \leq i \leq I_{t}}$ of $I_{t}$ points of $\left[\underline{v}_{t-1}, \bar{v}_{t-1}\right]$ and then for each $v_{t-1}^{i}$, we solve the above equation. Finally, this leads us to solve $M_{t} \times I_{t}$ systems of $d$ non-linear equations of $d$ unknowns:

$$
V_{t}\left(\theta_{t}^{*, i, j}, v_{t-1}^{i}, s_{t-1}^{j}, z_{t-1}^{j}\right)=0, \quad i=1, \cdots, I_{t}, \quad j=1, \cdots, M_{t} .
$$

For the sake of simplicity, we will temporarily drop $\left(s_{t-1}^{j}, z_{t-1}^{j}\right)$ in the above notations and try to emphasize how this system can be solved at each time step and for each nodes. Hence, we will denote $G_{t}(U)$ for $G_{t}\left(s_{t-1}^{j}, z_{t-1}^{j}, U_{t}\right), F_{t}(U)$ for $F_{t}\left(s_{t-1}^{j}, z_{t-1}^{j}, U\right)$ and by $v$ the value of the portfolio $v_{t-1}^{i}$. The local system (27) writes

$$
\mathbb{E}\left[G_{t}(U) l^{\prime}\left(-F_{t}(U)+\sum_{\ell=t+1}^{N} \theta_{\ell}^{*} \cdot \Delta S_{\ell}+\theta_{t}^{*} \cdot G_{t}(U)+v\right)\right]=0 .
$$

A natural method to estimate the (local) zero $\theta_{t}^{*}$ in (28) is to use a stochastic gradient algorithm derived from the (local) Lyapunov function $\theta \mapsto \mathbb{E}\left[l\left(-F_{t}(U)+\sum_{\ell=t+1}^{N} \theta_{\ell}^{*} \cdot \Delta S_{\ell}+\theta \cdot G_{t}(U)+v^{*}\right)\right]$. We make the following growth control of $l^{\prime}$, typically, $\left|l^{\prime}(x)\right| \leq C\left(1+e^{a x}\right), x \in \mathbb{R}, a, C>0$ and we suppose that $\mathbb{E}\left[G_{t}(U)^{4} e^{-4 a F_{t}(U)+4 a \sum_{\ell=t+1}^{N} \theta_{\ell}^{*} . \Delta S_{\ell}}\right]<+\infty$ and that there exists a function $\phi$ such that $\mathbb{E}\left[e^{4 a \theta \cdot G_{t}(U)}\right] \leq \phi(\theta), \theta \in \mathbb{R}^{d}$. These assumptions are satisfied if the price process $S$ is given by a discretized stochastic differential equation using an Euler scheme, so that in this case $G_{t}(U)$ is normally distributed. Now, one sets

$$
K\left(\theta, U, \Delta S_{t+1}, \cdots, \Delta S_{N}\right):=\frac{1}{1+\phi(\theta)} G_{t}(U) l^{\prime}\left(-F_{t}(U)+\sum_{\ell=t+1}^{N} \theta_{\ell}^{*} \cdot \Delta S_{\ell}+\theta \cdot G_{t}(U)+v\right)
$$


so that one may devise the following recursive scheme

$$
\theta_{n+1}=\theta_{n}-\gamma_{n+1} K\left(\theta_{n}, U_{n+1}, \Delta S_{t+1}^{n+1}, \cdots, \Delta S_{N}^{n+1}\right), \quad n \geq 0
$$

where $\left(U_{n+1}, \Delta S_{t+1}^{n+1}, \cdots, \Delta S_{N}^{n+1}\right)$ is an i.i.d. sequence of random vectors with the same distribution as $\left(U, \Delta S_{t+1}, \cdots, \Delta S_{N}\right)$, and $\left(\gamma_{n}\right)_{n \geq 1}$ is a positive deterministic sequence (decreasing to 0 ) satisfying (13). A straightforward application of Robbins-Monro's theorem yields the a.s. convergence of the sequence $\left(\theta_{n}\right)_{n \geq 1}$ toward the local $\theta_{t}^{*}$, as $n \rightarrow+\infty$.

As proposed in Section 3.2. one can also consider the deterministic counterpart of (29), i.e. a Newton-Raphson's algorithm built with $n$ samples, namely

$$
\theta_{i+1}=\theta_{i}-D k_{n}\left(\theta_{i}\right)^{-1} k_{n}\left(\theta_{i}\right), \quad i \geq 0
$$

where for $\theta \in \mathbb{R}^{d}$,

$$
\begin{aligned}
k_{n}(\theta) & :=\frac{1}{n} \sum_{j=1}^{n} G_{t}\left(U_{j}\right) l^{\prime}\left(-F_{t}\left(U_{j}\right)+\sum_{\ell=t+1}^{N} \theta_{\ell}^{*} \cdot \Delta S_{\ell}^{j}+\theta \cdot G_{t}\left(U_{j}\right)+v\right) \\
D k_{n}(\theta) & :=\frac{1}{n} \sum_{j=1}^{n} G_{t}\left(U_{j}\right) G_{t}\left(U_{j}\right)^{T} l^{\prime \prime}\left(-F_{t}\left(U_{j}\right)+\sum_{\ell=t+1}^{N} \theta_{\ell}^{*} \cdot \Delta S_{\ell}^{j}+\theta \cdot G_{t}\left(U_{j}\right)+v\right) .
\end{aligned}
$$

The main disavantage of Newton-Raphson's algorithm (30) compared to (29) is that it is known to converge only locally. However, in our numerical results, we observed that few steps of (30) are needed to estimate $\theta_{t}^{*}$ with a very good precision.

Remark 3.2. Let us note that in the case of the exponential loss $l(x):=e^{\lambda x}, \lambda>0, x \in \mathbb{R}$, we don't need to discretize the value of $V_{t-1}^{\theta}-\xi$ since the system (25) greatly simplifies and becomes:

$$
\mathbb{E}\left[\Delta S_{t} e^{\lambda\left(-H+\sum_{\ell=t+1}^{N} \theta_{\ell}^{*} . \Delta S_{\ell}+\theta_{t}^{*} . \Delta S_{t}\right)} \mid \mathcal{F}_{t-1}\right]=0, \text { a.s. }
$$

which can be written

$\mathbb{E}\left[G_{t}\left(s_{t-1}, z_{t-1}, U_{t}\right) e^{\lambda\left(-F_{t-1}\left(s_{t-1}, z_{t-1}, U_{t}\right)+\sum_{\ell=t+1}^{N} \theta_{\ell}^{*} \cdot \Delta S_{\ell}+\theta_{t}^{*} \cdot G_{t}\left(s_{t-1}, z_{t-1}, U_{t}\right)\right)}\right]_{\mid\left(s_{t-1}, z_{t-1}\right)=\left(S_{t-1}, Z_{t-1}\right)}=0$ a.s.

so that, one only needs to discretize the random vector $\left(S_{t-1}, Z_{t-1}\right)$ using Monte Carlo simulation or optimal vector quantization as presented above.

\section{Numerical tests}

In the examples below, we consider four different loss functions:

- $l_{\text {exp }}(x)=e^{x / 50}, x \in \mathbb{R}$ and $x_{0}=0$ which corresponds to the entropy risk measure with $\lambda=1 / 50$. Note that in this case, the shortfall risk can be computed by a classical Monte Carlo method using (44).

- $l_{p}(x)=x^{p} \mathbf{1}_{\{x \geq 0\}}, x \in \mathbb{R}, x_{0}=1$ and we take $p=2,3,4$. For those risk measures, the risk is estimated using the Robbins-Monro algorithm described in section 3.1 .

Using these loss functions, we will be interested in estimating the associated shortfall risks, a one step risk minimizing strategy and dynamic self-financed strategies. 


\begin{tabular}{|c|c|c|c|}
\hline$l$ & No hedging & One step hedging & $\theta^{*}$ \\
\hline$l_{\exp }$ & $-18.68 \pm 0.0241$ & $-21.32 \pm 0.02121$ & -14.70 \\
$l_{2}$ & $-1.95 \pm 0.00178$ & $-3.98 \pm 0.00768$ & -4.25 \\
$l_{3}$ & $-1.56 \pm 0.00098$ & $-2.65 \pm 0.00483$ & -2.32 \\
$l_{4}$ & $-1.40 \pm 0.00067$ & $-2.09 \pm 0.00324$ & -1.45 \\
\hline
\end{tabular}

Table 1: Shortfall risk estimation and One Step risk hedging of Spark Spread option

\subsection{Shortfall hedging of Spark Spread Option}

We consider a portfolio composed of an exchange option between gas and electricity (called Spark Spread) of maturity $T=1$ year. Since Electricity has very limited storage possibilities, the holder of this option hedges by trading only a gas forward contract of maturity $T$. The process $Z$ can be considered as the electricity spot price since it is observable on the energy market but cannot be used to set up hedging strategies. We choose to model the price $\left(S_{t}^{e}\right)_{0 \leq t \leq T}$ of the electricity spot contract and the price $\left(F_{t, T}^{g}\right)_{0 \leq t \leq T}$ of the gas forward contract of maturity $T$ by two independent geometric Brownian motions. Given that the price of the gas forward at maturity $T$ is $F^{g}(T, T)=S^{g}(T)$, the payoff $H$ of this contingent claim can be written

$$
H=\left(S_{T}^{e}-h_{R} S_{T}^{g}-C\right)_{+},
$$

where the heat rate $h_{R}=4 \mathrm{BTU} / \mathrm{kWh}$ (BTU: British Thermal Unit), the generation costs $C=$ $3 \$ / \mathrm{MWh}$, the two volatilities $\sigma_{g}=0.4, \sigma_{e}=0.8$ and the electricity and gas initial spot prices are $S_{0}^{e}=40 \$ / \mathrm{MWh}, S_{0}^{g}=3 \$ / \mathrm{MMBTU}$.

- Shortfall risk and One step hedging: A crude Monte Carlo gives $\mathbb{E}[L]=27.35 \pm 0.0436$ ( \pm 0.0436 corresponds to the width of the confidence interval at level 95\%) with a variance of 1487 after 3000000 trials. Concerning the Robbins-Monro algorithm (12), we define the step sequence by $\gamma_{n}=\frac{1}{n^{p}+100}$, with $p=\frac{3}{4}$. Note that for each case, the shortfall risk and optimal strategies are computed using the same pseudo-random number generator initialized with the same seed. Results about shortfall risk estimations and one step hedging are summarized in Table 1. The first column corresponds to the loss function. The second column corresponds to the risk without any hedging using the Robbins-Monro algorithm developed in section 3.1 with its confidence interval at level $95 \%$. The third column is the estimate of the shortfall risk with a one step risk minimization strategy and the fourth is the estimate of the optimal strategy $\theta^{*}$. Both estimates are obtained using the procedure developed in section 3.2. We observed that few steps are needed for the Newton-Raphson's algorithm (21) to converge, usually less than 10 steps is enough to estimate $\left(\xi^{*}, \theta^{*}\right)$ with a very good precision.

- Dynamic hedging: To complete this example, we experiment our algorithms to compute the optimal self-financed dynamic strategy. We consider three different values for the number of trading dates: $N=2$ (one trade per semester), $N=4$ (one trade per trimester) and $N=6$ (one trade each two months). All layers in the quantization tree of the random vector $\left(S_{t}^{e}, F_{t, T}^{g}\right)$ have the same size, i.e. $M=M_{1}=\cdots=M_{N}$, taking three possible values $M=5,10,20$. Note that actually at time step $t$, we consider an $M$-quantization grid of $\mathcal{N}\left(0, I_{2}\right):\left\{\left(g_{1}^{1}, g_{2}^{1}\right), \cdots,\left(g_{1}^{M}, g_{2}^{M}\right)\right\}$. A sharply optimized database of quantizers of standard univariate and multivariate Gaussian distributions is available on the web site www.quantize.maths-fi.com for download. Hence, we obtain a (nonoptimal) quantization grid of $\left(S_{t}^{e}, S_{t}^{g}\right)$, namely, for $j=1, \cdots, M$,

$$
s_{t}^{e, j}=S_{0}^{e} e^{-\frac{\sigma_{e}^{2}}{2} t+\sigma_{e} \sqrt{t} g_{1}^{j}}, \quad f_{t, T}^{g, j}=F_{0, T}^{g} e^{-\frac{\sigma_{g}^{2}}{2} t+\sigma_{g} \sqrt{t} g_{2}^{j}} .
$$


To determine the interval $\left[\underline{v}_{t}, \bar{v}_{t}\right]$, a natural idea is to replace the random variable $V_{t}^{\theta}-\xi$ by $\theta^{*} .\left(S_{t}-S_{0}\right)-\xi^{*}$ where $\left(\xi^{*}, \theta^{*}\right)$ is the solution of the one step risk hedging problem and to fix a (possibly non uniform) grid for this random variable. Hence in this example, we consider identical intervals $\left[\underline{v}_{t}, \bar{v}_{t}\right]=[\underline{v}, \bar{v}], t=2, \cdots, N-1$ and we set $\underline{v}=-15, \bar{v}=15$ with a uniform grid of 40 points. At each node of this grid (i.e. for each node of the quantization grid and each discrete point of the interval), we devise a Newton-Raphson's algorithm to estimate the optimal local strategy. We observed that few steps (less than five) are needed for this local optimization algorithm to converge. The results are summarized in Table 2, We can see that dynamic hedging significantly reduces the risk compared to one step hedging. However, one step hedging appears as a good "rough" hedge for an investor who does not want to hedge regularly. At each node of the dynamic programming backward algorithm, we observe that few iterations are needed to estimate the local strategy.

\begin{tabular}{|c|c|c|c|c|c|}
\hline $\mathrm{N}$ & $\mathrm{M}$ & $l_{\exp }$ & $l_{2}$ & $l_{3}$ & $l_{4}$ \\
\hline 2 & 5 & $-21.88 \pm 0.0211$ & $-4.65 \pm 0.0167$ & $-2.98 \pm 0.0174$ & $-2.26 \pm 0.0149$ \\
& 10 & $-22.09 \pm 0.0208$ & $-4.76 \pm 0.0197$ & $-3.12 \pm 0.0184$ & $-2.34 \pm 0.0169$ \\
& 20 & $-22.15 \pm 0.0205$ & $-4.78 \pm 0.0197$ & $-3.15 \pm 0.0172$ & $-2.38 \pm 0.0178$ \\
4 & 5 & $-22.20 \pm 0.0212$ & $-5.21 \pm 0.0203$ & $-3.25 \pm 0.0192$ & $-2.52 \pm 0.0197$ \\
& 10 & $-22.58 \pm 0.0201$ & $-5.50 \pm 0.0233$ & $-3.56 \pm 0.0154$ & $-2.65 \pm 0.0205$ \\
& 20 & $-22.68 \pm 0.0198$ & $-5.54 \pm 0.0211$ & $-3.64 \pm 0.0142$ & $-2.71 \pm 0.0188$ \\
6 & 5 & $-22.31 \pm 0.0197$ & $-5.61 \pm 0.0189$ & $-3.59 \pm 0.0166$ & $-2.58 \pm 0.0208$ \\
& 10 & $-22.76 \pm 0.0203$ & $-5.81 \pm 0.0224$ & $-3.76 \pm 0.0176$ & $-2.74 \pm 0.0208$ \\
& 20 & $-22.85 \pm 0.0196$ & $-5.84 \pm 0.0194$ & $-3.84 \pm 0.0194$ & $-2.77 \pm 0.0212$ \\
\hline
\end{tabular}

Table 2: Dynamic risk hedging of Spark Spread option

A natural application of our approach consists in the pricing of contingent claims in incomplete discrete time markets using the risk indifference principle. This recent approach has been proposed by [27, [47] and [33] among others. The buyer's (shortfall) risk indifference price $p^{b}$ is the initial payment that makes a person at time 0 risk indifferent between buying the contract with payoff $H$ and not buying the contract, with no initial payment. Hence, it is given by

$$
\inf _{\theta \in \mathcal{A}_{\mathcal{F}}} \rho\left(H-p^{b}-V_{N}^{\theta}\right)=\inf _{\theta \in \mathcal{A}_{\mathcal{F}}} \rho\left(-V_{N}^{\theta}\right)
$$

so that, using the invariance by translation property of $\rho$, we have

$$
p^{b}=\inf _{\theta \in \mathcal{A}_{\mathcal{F}}} \rho\left(-V_{N}^{\theta}\right)-\inf _{\theta \in \mathcal{A}_{\mathcal{F}}} \rho\left(H-V_{N}^{\theta}\right) .
$$

Consequently, we see that using the algorithm proposed in section 3.3. we are in position to fully characterize the buyer's risk indifference price $p^{b}$ since we are able to estimate $p^{b}$ and the corresponding minimizing strategies for each prices. Note that in this example, since the price of the gas forward contract is a $(\mathcal{F}, \mathbb{P})$-martingale we have $\inf _{\theta \in \mathcal{A}_{\mathcal{F}}} \rho\left(-V_{N}^{\theta}\right)=\rho(0)$ in our four cases. Hence, given that for $l=l_{\text {exp }}, \rho(0)=0$ and for $l=l_{2}, l_{3}, l_{4}, \rho(0)=-1$, the computation of the buyer's price is straightforward. Results are summarized in Table 3 ,

Remark 4.1. Let us note that we can do the same for the seller's (shortfall) risk indifference price $p^{s}$ which is the initial payment that makes a person at time 0 risk indifferent between selling the contract with payoff $H$ and not selling the contract, with no initial payment. Hence, it is clear that $p^{s}$ is given by

$$
p^{s}=\inf _{\theta \in \mathcal{A}_{\mathcal{F}}} \rho\left(V_{N}^{\theta}-H\right)-\inf _{\theta \in \mathcal{A}_{\mathcal{F}}} \rho\left(V_{N}^{\theta}\right)
$$




\begin{tabular}{|c|c|c|c|c|}
\hline Prices & $l_{\exp }$ & $l_{2}$ & $l_{3}$ & $l_{4}$ \\
\hline$p^{b}$ & 22.8 & 4.8 & 2.8 & 1.8 \\
\hline
\end{tabular}

Table 3: Buyer's risk indifference price of spark spread option with $M=10$ and $N=6$.

\begin{tabular}{|c|c|c|c|c|c|}
\hline & \multicolumn{2}{|c|}{ No hedging } & \multicolumn{3}{c|}{ One step hedging } \\
\hline$l$ & $d=2$ & $d=3$ & $\left(d, d^{\prime}\right)=(2,1)$ & $\left(d, d^{\prime}\right)=(3,1)$ & $\left(d, d^{\prime}\right)=(3,2)$ \\
\hline$l_{\exp }$ & $-2.15 \pm 0.0167$ & $-1.55 \pm 0.0128$ & $-2.23 \pm 0.0145$ & $-1.58 \pm 0.0157$ & $-1.61 \pm 0.0105$ \\
$l_{2}$ & $-1.18 \pm 0.00130$ & $-1.15 \pm 0.00117$ & $-1.29 \pm 0.0324$ & $-1.21 \pm 0.0254$ & $-1.28 \pm 0.0204$ \\
$l_{3}$ & $-1.12 \pm 0.00083$ & $-1.10 \pm 0.00076$ & $-1.18 \pm 0.0254$ & $-1.13 \pm 0.0230$ & $-1.17 \pm 0.0196$ \\
$l_{4}$ & $-1.09 \pm 0.00061$ & $-1.08 \pm 0.00056$ & $-1.13 \pm 0.0232$ & $-1.10 \pm 0.0198$ & $-1.12 \pm 0.0155$ \\
\hline
\end{tabular}

Table 4: Shortfall risk estimation and One Step risk hedging of Basket options with $d=2,3$, $d^{\prime}=1,2, T=1, S_{0}^{i}=50, \sigma^{i}=0.3, w_{i}=1 / d, i=1, \cdots, d, K=55$.

Hence, we see that the seller's risk indifference price can also be computed using the procedures developed in 3.3 .

\subsection{Shortfall hedging of Basket option}

We consider basket options with payoffs given by $\left(\sum_{i}^{d} w_{i} S_{T}^{i}-K\right)_{+}$where $\left(w_{1}, \cdots, w_{d}\right)$ is the vector of weights, $K$ denotes the strike, $T$ is the maturity and $S_{T}^{i}$ is the price at maturity of the ith asset. We assume that each of the $d$ assets has a price given by a Black-Scholes model driven by the vector of independent Brownian motions $W=\left(W_{t}^{1}, \cdots, W_{t}^{d}, t \geq 0\right)$,

$$
S_{t}^{i}=S_{0}^{i} e^{\left(r-\frac{\sigma_{i}^{2}}{2}\right) t+\sigma_{i} W_{t}^{i}} \stackrel{\mathcal{L}}{=} S_{0}^{i} e^{\left(r-\frac{\sigma_{i}^{2}}{2}\right) t+\sigma_{i} \sqrt{t} Z_{i}}, \quad S_{0}=\left(S_{0}^{1}, \cdots, S_{0}^{d}\right),
$$

where $Z=\left(Z_{1}, \cdots, Z_{d}\right)$ is a Gaussian vector of size $d$. The source of incompleteness of the market comes from the fact that there are some assets that are observable but not available for trade. This situation may happen if the transaction costs of some assets which appear in the basket option are too high. We suppose that the investor trades only the first $d^{\prime}(<d)$ assets, i.e. he uses them to build our optimal risk minimizing strategies. We compute the shortfall risks and the optimal dynamic strategies for different values of $d$ and $d^{\prime}$. The (non-optimal) quantization grids of $\left(S_{t}^{i}\right)_{1 \leq i \leq d}$ are calculated from the optimal quantization grid of $\left(Z_{i}\right)_{1 \leq i \leq d}$ and have the same size $M=10$. The number of trading dates $N=4$ is fixed and the number of Monte Carlo simulations is 300,000 in every case. Following the idea proposed in 4.1, we consider identical intervals $\left[\underline{v}_{t}, \bar{v}_{t}\right]=[\underline{v}, \bar{v}]$, $t=2, \cdots, N-1$ and we set $\underline{v}=-5$ and $\bar{v}=5$ with a uniform grid of 10 points. Note that for each case, the shortfall risk and optimal strategies are computed using the same pseudo-random number generator initialized with the same seed. Results are summarized in Table 4 for the shortfall risk computation and the one step hedging. Results are summarized in Table 5 for the dynamic case. One deduces easily the buyer's risk indifference prices of each Basket option using those results. Once again, we observe that a one step hedging is a good hedge and dynamic hedging refines this first result. As expected, using 2 assets rather than one provides a better reduction of the risk.

\subsection{Shortfall hedging of gas consumption}

At time $T=1$ (year), an energy provider buys on the gas market a quantity $C_{T}$ of gas at price $F_{T, T}^{g}=S_{T}^{g}$ and sells it to consumers at a fixed price $K=11 € / \mathrm{MWh}$. The quantity $C_{T}$ denotes the 


\begin{tabular}{|c|c|c|c|}
\hline & \multicolumn{3}{|c|}{ Dynamic hedging } \\
\hline$l$ & $\left(d, d^{\prime}\right)=(2,1)$ & $\left(d, d^{\prime}\right)=(3,1)$ & $\left(d, d^{\prime}\right)=(3,2)$ \\
\hline$l_{\exp }$ & $-2.26 \pm 0.0072$ & $-1.60 \pm 0.0113$ & $-1.63 \pm 0.0052$ \\
$l_{2}$ & $-1.41 \pm 0.0039$ & $-1.25 \pm 0.0026$ & $-1.40 \pm 0.0037$ \\
$l_{3}$ & $-1.24 \pm 0.0024$ & $-1.15 \pm 0.0016$ & $-1.23 \pm 0.0026$ \\
$l_{4}$ & $-1.16 \pm 0.0018$ & $-1.12 \pm 0.0011$ & $-1.15 \pm 0.0021$ \\
\hline
\end{tabular}

Table 5: Dynamic risk hedging of Basket options with $d=2,3, d^{\prime}=1,2, T=1, S_{0}^{i}=50, \sigma^{i}=0.3$, $w_{i}=1 / d, i=1, \cdots, d, K=55, M=10, N=4$.

consumption at time $T$ and is equal to $C_{T}=a-b Z_{T}$, where $a=10 \mathrm{Mwh}$ and $b=0.3 \mathrm{MWh} /{ }^{\circ} \mathrm{Cand}$ $Z_{T}$ is the temperature at time $T$. The temperature is modeled by a Gaussian Ornstein-Uhlenbeck process so that it is given by

$$
Z_{T}=e^{-\lambda T} Z_{0}+m\left(1-e^{-\lambda T}\right)+\sigma_{Z} \sqrt{\frac{1-e^{-2 \lambda T}}{2 \lambda}} G_{1}
$$

with $Z_{0}=11^{\circ} \mathrm{C}, \lambda=0.02, m=11^{\circ} \mathrm{C}, \sigma_{Z}=6^{\circ} \mathrm{C}$ and $G_{1} \sim \mathcal{N}(0,1)$. The gas forward price of maturity $T$ is modeled as a geometric Brownian motion with $F_{0, T}^{g}=11 € / \mathrm{MWh}$ and the Brownian motion of gas spot price is correlated with the one of the temperature, $\rho=-0.2$, namely

$$
F_{t, T}^{g}=F_{0, T}^{g} e^{-\frac{\sigma_{T}^{2}}{2} t+\sigma_{T} \sqrt{t}\left(\rho G_{1}+\sqrt{1-\rho^{2}} G_{2}\right)},
$$

where $\sigma_{T}=0.4, G_{2} \sim \mathcal{N}(0,1)$, and is independent of $G_{1}$. Consequently, the loss suffered by the energy provider at time $T$ is given by

$$
L=\left(F_{T, T}^{g}-K\right) C_{T} .
$$

The energy provider uses a self-financed static strategy based on the gas forward price of maturity $T$ in order to reduce its shortfall risk at time $t_{0}=0$ and wants to compare the shortfall risk and the shortfall (one-step and dynamic) hedges. A crude Monte Carlo gives $\mathbb{E}[L]=1.57 \pm 0.12$ with a variance of 1088 after 300000 trials. The number of Monte Carlo simulations used in all procedures is set to 300000 and the pseudo-random number generator is initialized with the same seed. Concerning dynamic hedging strategies, the number of trading dates $N=4$ is fixed. We obtain a (non-optimal) quantization grid of $\left(F_{t, T}^{g}, Z_{t}\right)$ of size $M=10$, namely, for $j=1, \cdots, M$,

$$
f_{t, T}^{g, j}=F_{0, T}^{g} e^{-\frac{\sigma_{T}^{2}}{2} t+\sigma_{T} \sqrt{t}\left(\rho g_{1}^{j}+\sqrt{1-\rho^{2}} g_{2}^{j}\right)}, \quad z_{t}^{j}=e^{-\lambda t} Z_{0}+m\left(1-e^{-\lambda t}\right)+\sigma_{Z} \sqrt{\frac{1-e^{-2 \lambda t}}{2 \lambda}} g_{1}^{j} .
$$

Concerning the discretization of the portfolio values, as explained before we consider identical intervals $\left[\underline{v}_{t}, \bar{v}_{t}\right]=[\underline{v}, \bar{v}], t=2, \cdots, N-1$ and we set $\underline{v}=-50$ and $\bar{v}=50$ with a uniform grid of 10 points. Results are summarized in Table 6. Surprisingly, we see that the entropy risk becomes negative when we use a one step or a dynamic hedging strategy. In this example, one step hedging greatly reduces the risk of this position and a dynamic hedges significantly improves this rough hedge. For power risk measures, dynamic strategies are able to divide the risk by a factor between 2 and 3.

\section{References}

[1] P. Artzner, F. Delbaen, J.-M. Eber, and D. Heath. Coherent measures of risk. Math. Finance, 9(3):203$228,1999$. 


\begin{tabular}{|c|c|c|c|c|}
\hline$l$ & No hedging & One step hedging & $\theta^{*}$ & Dynamic Hedging \\
\hline$l_{\exp }$ & $6.61 \pm 0.09$ & $-0.94 \pm 0.03$ & 6.8 & $-1.14 \pm 0.01$ \\
$l_{2}$ & $34.24 \pm 0.14$ & $14.64 \pm 0.33$ & 5.8 & $11.29 \pm 0.27$ \\
$l_{3}$ & $53.30 \pm 0.57$ & $28.53 \pm 1.71$ & 4.9 & $22.40 \pm 1.68$ \\
$l_{4}$ & $66.43 \pm 1.47$ & $41.64 \pm 2.35$ & 4.5 & $31.93 \pm 2.90$ \\
\hline
\end{tabular}

Table 6: Risk hedging of gas consumption with $F_{0, T}^{g}=11 € / \mathrm{MWh}, K=11 € / \mathrm{MWh}, a=10 \mathrm{Mwh}$ and $b=0.3 \mathrm{MWh} /{ }^{\circ} \mathrm{C}, Z_{0}=11^{\circ} \mathrm{C}, \lambda=0.02, m=11^{\circ} \mathrm{C}, \sigma_{Z}=6^{\circ} \mathrm{C}, \sigma_{T}=0.4, N=4$ and $M=10$.

[2] O. Bardou, N. Frikha, and G. Pagès. Computing VaR and CVaR using stochastic approximation and adaptive unconstrained importance sampling. Monte Carlo Methods Appl., 15(3):173-210, 2009.

[3] O. Bardou, N. Frikha, and G. Pagès. CVaR hedging using quantization based stochastic approximation algorithm. Preprint, LPMA, 2010.

[4] P. Barrieu and N. El Karoui. Optimal derivatives design under dynamic risk measures. Mathematics of Finance, Contemporary Mathematics 351, American Mathematical Society, pages 13-25, 2004.

[5] N. Bellamy and M. Jeanblanc. Incompleteness of markets driven by a mixed diffusion. Finance Stoch., $4(2): 209-222,2000$.

[6] A. Benveniste, M. Métivier, and P. Priouret. Adaptive algorithms and stochastic approximations, volume 22 of Applications of Mathematics (New York). Springer-Verlag, Berlin, 1990. Translated from the French by Stephen S. Wilson.

[7] J. Cvitanić and I. Karatzas. On dynamic measures of risk. Finance Stoch., 3(4):451-482, 1999.

[8] J. Cvitanić, H. Pham, and N. Touzi. Super-replication in stochastic volatility models under portfolio constraints. J. Appl. Probab., 36(2):523-545, 1999.

[9] C. Dellacherie and P.-A. Meyer. Probabilités et potentiel. Hermann, Paris, 1975. Chapitres I à IV, Édition entièrement refondue, Publications de l'Institut de Mathématique de l'Université de Strasbourg, No. XV, Actualités Scientifiques et Industrielles, No. 1372.

[10] J. E. Dennis, Jr. and R. B. Schnabel. Numerical methods for unconstrained optimization and nonlinear equations, volume 16 of Classics in Applied Mathematics. Society for Industrial and Applied Mathematics (SIAM), Philadelphia, PA, 1996. Corrected reprint of the 1983 original.

[11] D. Duffie and H. R. Richardson. Mean-variance hedging in continuous time. Ann. Appl. Probab., $1(1): 1-15,1991$.

[12] M. Duflo. Algorithmes stochastiques, volume 23 of Mathématiques $\& 3$ Applications (Berlin) [Mathematics Es Applications]. Springer-Verlag, Berlin, 1996.

[13] M. Duflo. Iterative random models. transl. from French,, 1997.

[14] E. Eberlein and J. Jacod. On the range of option prices. Finance Stoch., 1(2):131-140, 1997.

[15] N. El Karoui and M.-C. Quenez. Dynamic programming and pricing of contingent claims in an incomplete market. SIAM J. Control Optim., 33(1):29-66, 1995.

[16] I. Evstigneev. Measurable selection and dynamic programming. Mathematics of Operations Research, $1(3): 267-272,1976$.

[17] D. Filipovic and G. Svindland. The canonical model space for law-invariant convex risk measures is $l^{1}$. Mathematical Finance, 2009.

[18] H. Föllmer and Y. Kabanov. Optional decomposition and lagrange multipliers. Finance Stoch., 2:69-81, 1998.

[19] H. Föllmer and P. Leukert. Quantile hedging. Finance Stoch., 3(3):251-273, 1999. 
[20] H. Föllmer and P. Leukert. Efficient hedging: cost versus shortfall risk. Finance Stoch., 4(2):117-146, 2000 .

[21] H. Föllmer and A. Schied. Convex measures of risk and trading constraints. Finance Stoch., 6(4):429$447,2002$.

[22] H. Föllmer and A. Schied. Stochastic finance. Walter de Gruyter \& Co., Berlin, extended edition, 2011. An introduction in discrete time.

[23] M. Frittelli and E. Rosazza Gianin. Putting order in risk measures. Journal of Banking and Finance, 26:1473-1486, 2002.

[24] J. Jacod and A. Shiryayev. Local martingales and the fundamental asset pricing theorems in the discrete-time case. Finance and Stochastics, 2:259-273, 1998.

[25] B. Jourdain and J. Lelong. Robust adaptive importance sampling for normal random vectors. Ann. Appl. Probab., 19(5):1687-1718, 2009.

[26] M. Kaina and L. Rüschendorf. On convex risk measures on $l^{p}$-spaces. Mathematical Methods in Operations Research (MMR), 69:475-495, 2009.

[27] S. Klöppel and M. Schweizer. Dynamic indifference valuation via convex risk measures. Math. Finance, 17:599-627, 2007.

[28] D. Kramkov. Optional decomposition of supermartingales and hedging contingent claims in incomplete security markets. Probability Theory and Related Fields, 105:459-479, 1996.

[29] H. Kushner and G. Yin. Stochastic Approximation and Recursive Algorithms and Applications. Springer, 2003.

[30] J. Laurent and H. Pham. Dynamic programming and mean-variance hedging. Finance and Stochastics, 23:83-110, 1999.

[31] D. G. Luenberger and Y. Ye. Linear and nonlinear programming. International Series in Operations Research \& Management Science, 116. Springer, New York, third edition, 2008.

[32] P. Monat and C. Stricker. Föllmer-schweizer decomposition and mean-variance hedging of general claims. Annals of Probability, 23:605-628, 1995.

[33] B. Oksendal and A. Sulem. Risk indifference pricing in jump diffusion markets. Math. Finance, 19:229$239,2009$.

[34] G. Pagès. A space vector quantization method for numerical integration. J. Computational and Applied Math., 89:1-38, 1997.

[35] H. Pham. Dynamic $l^{p}$-hedging in discrete time under cone constraints. SIAM Journal on Control and Optimization, 38(3):665-682, 2000.

[36] B. T. Polyak and A. B. Juditsky. Acceleration of stochastic approximation by averaging. SIAM J. Control Optim., 30(4):838-855, 1992.

[37] M. Rásonyi and L. Stettner. On utility maximization in discrete-time financial market models. Annals of Applied Probability, 15(2):1367-1395, 2005.

[38] T. Rheinländer and M. Schweizer. On $l^{2}$-projections on a space of stochastic integrals. Annals of Probability, 25:1810-1831, 1997.

[39] R. Rockafellar and S. Uryasev. Optimization of conditional value-at-risk. Journal of Risk, 2:493-517, 2000 .

[40] L. C. G. Rogers. Equivalent martingale measures and no-arbitrage. Stochastics Stochastics Rep., 51(12):41-49, 1994.

[41] D. Ruppert. Stochastic approximation. In Handbook of sequential analysis, volume 118 of Statist. Textbooks Monogr., pages 503-529. Dekker, New York, 1991. 
[42] W. Schachermayer. A Hilbert space proof of the fundamental theorem of asset pricing in finite discrete time. Insurance Math. Econom., 11(4):249-257, 1992.

[43] M. Schäl. On quadratic cost criteria for option hedging. Math. Oper. Res., 19(1):121-131, 1994.

[44] M. Schäl. Martingale measures and hedging for discrete-time financial markets. Math. Oper. Res., 24(2):509-528, 1999.

[45] M. Schweizer. Variance-optimal hedging in discrete time. Math. Oper. Res., 20(1):1-32, 1995.

[46] M. Schweizer. Approximation pricing and the variance-optimal martingale measure. Ann. Probab., 24(1):206-236, 1996.

[47] M. Xu. Risk measure pricing and hedging in incomplete markets. Ann. of Finance, 2:51-71, 2005.

\section{Appendix}

\subsection{Proof of Theorem 2.5}

We proceed by backward induction.

Step 1: At step $N-1$, we solve the following stochastic control problem

$$
\varphi_{N-1}\left(\xi, \theta_{1}, \cdots, \theta_{N-1}\right):=\underset{\theta_{N} \in \Theta_{N-1}}{\operatorname{essinf}} \mathbb{E}\left[l\left(-H+V_{N}^{\theta}-\xi\right) \mid \mathcal{F}_{N-1}\right], \text { a.s. }
$$

Let $w \in \Omega$ and $\left(\theta_{1}, \cdots, \theta_{N-1}\right) \in \Theta_{0} \times \cdots \times \Theta_{N-2}$. By virtue of Proposition 2.4 the function defined by $\theta_{N}(w) \mapsto \mathbb{E}\left[l\left(-H+\theta_{N} . \Delta S_{N}+V_{N-1}^{\theta}-\xi\right) \mid \mathcal{F}_{N-1}\right](w)=f\left(\xi, V_{N-1}^{\theta}(w), \theta_{N}(w), w\right)$, where $f$ is the associated regular conditional expectation, is l.s.c., convex and coercive. Consequently, there always exists $\bar{\theta} \in \mathbb{R}^{d}$ such that $f\left(\xi, V_{N-1}^{\theta}(w), \bar{\theta}, w\right)=\inf _{\phi \in \mathbb{R}^{d}} f\left(\xi, V_{N-1}^{\theta}(w), \phi, w\right)$. It is clear that $(\xi, x, \theta, w) \mapsto f(\xi, x, \theta, w)$ is an $\mathcal{B}(\mathbb{R}) \otimes \mathcal{B}(\mathbb{R}) \otimes \mathcal{B}\left(\mathbb{R}^{d}\right) \otimes \mathcal{F}_{N-1}$-measurable function and $(\xi, x, w) \mapsto \inf _{\phi \in \mathbb{R}^{d}} f(\xi, x, \phi, w)$ is an $\mathcal{B}(\mathbb{R}) \otimes \mathcal{B}(\mathbb{R}) \otimes$ $\mathcal{F}_{N-1}$-measurable function. Hence, the set

$$
A=\left\{(w, \xi, x, \theta) \in \Omega \times \mathbb{R} \times \mathbb{R} \times \mathbb{R}^{d}: f(\xi, x, \theta, w)=\inf _{\phi \in \mathbb{R}^{d}} f(\xi, x, \phi, w)\right\}
$$

is $\mathcal{F}_{N-1} \otimes \mathcal{B}(\mathbb{R}) \otimes \mathcal{B}(\mathbb{R}) \otimes \mathcal{B}\left(\mathbb{R}^{d}\right)$-measurable. Its projection on $\Omega \times \mathbb{R} \times \mathbb{R}$ is equal to $\Omega \times \mathbb{R} \times \mathbb{R}$. Then Lemma 2.3 yields an $\mathcal{B}(\mathbb{R}) \otimes \mathcal{B}(\mathbb{R}) \otimes \mathcal{F}_{N-1}$ measurable vector $\theta_{N}^{*}$ such that

$$
f\left(\xi, x, \theta_{N}^{*}(\xi, x, w), w\right)=\inf _{\phi \in \mathbb{R}^{d}} f(\xi, x, \phi, w)
$$

for $\mathbb{P}$-almost all $w \in \Omega, \lambda$-almost all $\xi \in \mathbb{R}$ and $\lambda$-almost all $x \in \mathbb{R}$. Consequently, the random variable $\theta_{N}^{*}\left(\xi, V_{N-1}^{\theta}(),.\right): \Omega \rightarrow \mathbb{R}^{d}$ is $\mathcal{F}_{N-1}$-measurable and provides the optimal strategy at step $N-1$ satisfying

$$
\varphi_{N-1}\left(\xi, \theta_{1}, \cdots, \theta_{N-1}\right)=\mathbb{E}\left[l\left(-H+\theta_{N}^{*} . \Delta S_{N}+V_{N-1}^{\theta}-\xi\right) \mid \mathcal{F}_{N-1}\right], \quad \text { a.s. }
$$

Step 2: Now, suppose that at step $t$, we have constructed $\left(\theta_{t+1}^{*}, \cdots, \theta_{N}^{*}\right) \in \Theta_{t} \times \cdots \times \Theta_{N-1}$ such that

$$
\begin{aligned}
\varphi_{t}\left(\xi, \theta_{1}, \cdots, \theta_{t}\right) & =\underset{\theta_{t+1} \in \Theta_{t}}{\operatorname{essinf}} \mathbb{E}\left[\varphi_{t+1}\left(\xi, \theta_{1}, \cdots, \theta_{t+1}, w\right) \mid \mathcal{F}_{t}\right] \\
& =\mathbb{E}\left[l\left(-H+\sum_{\ell=t+1}^{N} \theta_{\ell}^{*} \cdot \Delta S_{\ell}-V_{t}^{\theta}-\xi\right) \mid \mathcal{F}_{t}\right], \\
& =\underset{\left(\theta_{t+1}, \cdots, \theta_{N}\right) \in \Theta_{t} \times \cdots \times \Theta_{N-1}}{\operatorname{essinf}} \mathbb{E}\left[l\left(-H+V_{N}^{\theta}-\xi\right) \mid \mathcal{F}_{t}\right] .
\end{aligned}
$$

Hence, it is clear that the function $\varphi_{t-1}$ satisfies the following equalities

$$
\begin{aligned}
\varphi_{t-1}\left(\xi, \theta_{1}, \cdots, \theta_{t-1}\right) & =\underset{\theta_{t} \in \Theta_{t-1}}{\operatorname{essinf}} \mathbb{E}\left[\varphi_{t}\left(\xi, \theta_{1}, \cdots, \theta_{t}\right) \mid \mathcal{F}_{t-1}\right] \\
& =\underset{\theta_{t} \in \Theta_{t-1}}{\operatorname{essinf}} \mathbb{E}\left[\mathbb{E}\left[l\left(-H+\sum_{\ell=t+1}^{N} \theta_{\ell}^{*} \cdot \Delta S_{\ell}-V_{t}^{\theta}-\xi\right) \mid \mathcal{F}_{t}\right] \mid \mathcal{F}_{t-1}\right], \\
& =\underset{\left(\theta_{t}, \cdots, \theta_{N}\right) \in \Theta_{t-1} \times \cdots \times \Theta_{N-1}}{\operatorname{essinf}} \mathbb{E}\left[l\left(-H+V_{N}^{\theta}-\xi\right) \mid \mathcal{F}_{t-1}\right] .
\end{aligned}
$$


Proposition 2.4 implies that the function $\left(\theta_{t}, \cdots, \theta_{N}\right) \mapsto \mathbb{E}\left[l\left(-H+V_{N}^{\theta}-\xi\right) \mid \mathcal{F}_{t-1}\right]$ is l.s.c., convex and coercive so that for every $c<+\infty$, the following set

$$
\begin{aligned}
\left\{\theta_{t}: \underset{\left(\theta_{t+1}, \cdots, \theta_{N}\right) \in \Theta_{t} \times \cdots \times \Theta_{N-1}}{\operatorname{essinf}} \mathbb{E}\left[l\left(-H+V_{N}^{\theta}-\xi\right) \mid \mathcal{F}_{t-1}\right](w) \leq c\right\}= \\
\operatorname{Proj}_{1}\left\{\left(\theta_{t}, \cdots, \theta_{N}\right): \mathbb{E}\left[l\left(-H+V_{N}^{\theta}-\xi\right) \mid \mathcal{F}_{t-1}\right](w) \leq c\right\}
\end{aligned}
$$

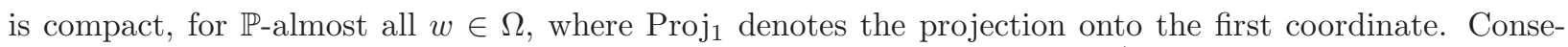
quently, the function $\theta_{t} \mapsto \operatorname{ess}_{\inf }\left(\theta_{t+1}, \cdots, \theta_{N}\right) \in \Theta_{t} \times \cdots \times \Theta_{N-1} \mathbb{E}\left[l\left(-H+V_{N}^{\theta}-\xi\right) \mid \mathcal{F}_{t-1}\right]$ is l.s.c. It is clear that it is a convex function. The coercivity follows using similar arguments than those used in the proof of Proposition 2.4. Hence, following the arguments used in Step 1 yields an $\mathcal{B}(\mathbb{R}) \otimes \mathcal{B}(\mathbb{R}) \otimes \mathcal{F}_{t-1}$ measurable vector $\theta_{t}^{*}:=\theta_{t}^{*}\left(\xi, V_{t-1}^{\theta}(w), w\right)$ such that

$$
\begin{aligned}
\varphi_{t-1}\left(\xi, \theta_{1}, \cdots, \theta_{t-1}\right) & =\mathbb{E}\left[\varphi_{t}\left(\xi, \theta_{1}, \cdots, \theta_{t}^{*}\right) \mid \mathcal{F}_{t-1}\right] \\
& =\mathbb{E}\left[l\left(-H+\sum_{\ell=t}^{N} \theta_{\ell}^{*} \cdot \Delta S_{\ell}+V_{t-1}^{\theta}-\xi\right) \mid \mathcal{F}_{t-1}\right] \\
& =\underset{\left(\theta_{t}, \cdots, \theta_{N}\right) \in \Theta_{t-1} \times \cdots \times \Theta_{N-1}}{\operatorname{essinf}} \mathbb{E}\left[l\left(-H+V_{N}^{\theta}-\xi\right) \mid \mathcal{F}_{t-1}\right],
\end{aligned}
$$

for $\mathbb{P}$-almost all $w \in \Omega$ and $\lambda$-almost all $\xi \in \mathbb{R}$. This concludes the proof.

\subsection{Proof of Theorem 2.6}

We proceed by backward induction.

Step 1: For $t=N$, we want to derive the first order condition associated to the one-step stochastic control problem

$$
\underset{\theta_{N} \in \Theta_{N-1}}{\operatorname{essinf}} \mathbb{E}\left[l\left(-H+\theta_{N} \cdot \Delta S_{N}+V_{N-1}^{\theta}-\xi\right) \mid \mathcal{F}_{N-1}\right] .
$$

To this end, we prove that the function $G_{N-1}:(x, \theta) \mapsto \mathbb{E}\left[g_{N}\left(H, \Delta S_{N}, x, \theta, \xi\right) \mid \mathcal{F}_{N-1}\right]$, with $g_{N}\left(H, \Delta S_{N}, x, \theta, \xi\right):=$ $l\left(-H+\theta . \Delta S_{N}+x-\xi\right)$, is differentiable and compute its gradient. In order to justify the formal differentiation, we compute the local gradient which is clearly given by

$$
\begin{aligned}
& \nabla_{x} g_{N}\left(H, \Delta S_{N}, x, \theta, \xi\right)=l^{\prime}\left(-H+\theta \cdot \Delta S_{N}+x-\xi\right) \\
& \nabla_{\theta} g_{N}\left(H, \Delta S_{N}, x, \theta, \xi\right)=\Delta S_{N} l^{\prime}\left(-H+\theta \cdot \Delta S_{N}+x-\xi\right) .
\end{aligned}
$$

Using assumption $2.2(i)$, we clearly have

$$
\begin{aligned}
\left|\nabla_{(x, \theta)} g_{N}\left(H, \Delta S_{N}, x, \theta, \xi\right)\right| & \leq\left(\left|\Delta S_{N}\right|+1\right) l^{\prime}\left(-H+\theta \cdot \Delta S_{N}+x-\xi\right) \\
& \leq C\left(\left|\Delta S_{N}\right|+1\right) l\left(-H+\theta \cdot \Delta S_{N}+x-\xi\right)
\end{aligned}
$$

consequently, in order to justify the local differentiation under (conditional) expectation, we prove that for every $R>0$, the family of random variables

$$
\left\{\left(\left|\Delta S_{N}\right|+1\right) l\left(-H+\theta . \Delta S_{N}+x-\xi\right) ;(x, \theta) \in \bar{B}_{1}(0, R) \times \bar{B}_{d}(0, R)\right\}
$$

is (conditinally) uniformly integrable. This may be justified as follows. Assumption 2.2 (ii), (iii) and Cauchy-Schwarz's inequality implies that for $\alpha>1$,

$$
\begin{aligned}
\sup _{(x, \theta) \in \bar{B}_{1}(0, R) \times \bar{B}_{d}(0, R)} & \mathbb{E}\left[\left(\left|\Delta S_{N}\right|+1\right)^{\alpha} l^{\alpha}\left(-H+\theta . \Delta S_{N}+x-\xi\right) \mid \mathcal{F}_{N-1}\right] \leq C \mathbb{E}\left[\left(\left|\Delta S_{N}\right|^{2 \alpha}+1\right) \mid \mathcal{F}_{N-1}\right]^{1 / 2} \\
& \times \sup _{(x, \theta) \in \bar{B}_{1}(0, R) \times \bar{B}_{d}(0, R)} \mathbb{E}\left[l^{2 \alpha}\left(-H+\theta . \Delta S_{N}+x-\xi\right) \mid \mathcal{F}_{N-1}\right]^{1 / 2}<+\infty \text { a.s. }
\end{aligned}
$$

Hence the function $G_{N-1}$ is continuously differentiable and we have

$$
\nabla_{\left(x, \theta_{N}\right)} G_{N-1}\left(V_{N-1}^{\theta}, \theta_{N}\right)=\mathbb{E}\left[\nabla_{(x, \theta)} g_{N}\left(H, \Delta S_{N}, V_{N-1}^{\theta}, \theta_{N}\right) \mid \mathcal{F}_{N-1}\right]
$$


and $\theta_{N}^{*}$ satisfies

$$
\mathbb{E}\left[\Delta S_{N} l^{\prime}\left(-H+\theta_{N}^{*} \cdot \Delta S_{N}+V_{N-1}^{\theta}-\xi\right) \mid \mathcal{F}_{N-1}\right]=0, \text { a.s. }
$$

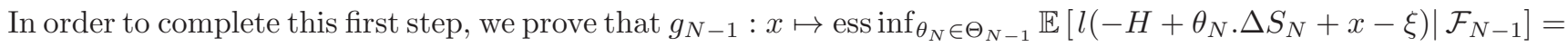

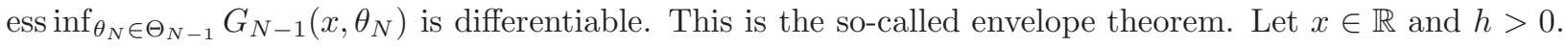
The optimality of $\theta_{N}^{*}(x)$ implies that

$$
g_{N-1}(x \pm h)-g_{N-1}(x) \leq G_{N-1}\left(x \pm h, \theta_{N}^{*}(x)\right)-G_{N-1}\left(x, \theta_{N}^{*}(x)\right),
$$

so that using the convexity of $g_{N-1}$, we obtain

$$
\begin{aligned}
& \frac{G_{N-1}\left(x-h, \theta_{N}^{*}(x)\right)-G_{N-1}\left(x, \theta_{N}^{*}(x)\right)}{-h} \leq \frac{g_{N-1}(x-h)-g_{N-1}(x)}{-h} \\
& \quad \leq \frac{g_{N-1}(x+h)-g_{N-1}(x)}{h} \leq \frac{G_{N-1}\left(x+h, \theta_{N}^{*}(x)\right)-G_{N-1}\left(x, \theta_{N}^{*}(x)\right)}{h} .
\end{aligned}
$$

Hence, letting $h \rightarrow 0$ and using the differentiability of $G_{N-1}$ yields the result. The continuous differentiability of $G_{N-1}$ assures that one can plug $V_{N-1}^{\theta}$ into the gradient of $G_{N-1}$.

Step 2: At step $t$, we suppose that the function

$$
x \mapsto \underset{\left(\theta_{t+1}, \cdots, \theta_{N}\right) \in \Theta_{t} \times \cdots \times \Theta_{N-1}}{\operatorname{essinf}} \mathbb{E}\left[l\left(-H+\sum_{\ell=t+1}^{N} \theta_{\ell} \cdot \Delta S_{\ell}+x-\xi\right) \mid \mathcal{F}_{t}\right]
$$

is differentiable with a derivative given by $\mathbb{E}\left[l^{\prime}\left(-H+\sum_{\ell=t+1}^{N} \theta_{\ell}^{*} \cdot \Delta S_{\ell}+x-\xi\right) \mid \mathcal{F}_{t}\right]$ and we want to derive the first order condition associated to the stochastic control problem

$$
\underset{\theta_{t} \in \Theta_{t-1}}{\operatorname{essinf}} \mathbb{E}\left[\underset{\left(\theta_{t+1}, \cdots, \theta_{N}\right) \in \Theta_{t} \times \cdots \times \Theta_{N-1}}{\operatorname{essinf}} \mathbb{E}\left[l\left(-H+\sum_{\ell=t+1}^{N} \theta_{\ell} \cdot \Delta S_{\ell}+V_{t}^{\theta}-\xi\right) \mid \mathcal{F}_{t}\right] \mid \mathcal{F}_{t-1}\right] .
$$

The proof uses similar arguments as those used in Step 1. We introduce the function $G_{t-1}$ defined for every $(x, \theta) \in \mathbb{R} \times \mathbb{R}^{d}$ by

$$
G_{t-1}(x, \theta)=\mathbb{E}\left[\underset{\left(\theta_{t+1}, \cdots, \theta_{N}\right) \in \Theta_{t} \times \cdots \times \Theta_{N-1}}{\operatorname{sssinf}} \mathbb{E}\left[l\left(-H+\sum_{\ell=t+1}^{N} \theta_{\ell} \cdot \Delta S_{\ell}+\theta \cdot \Delta S_{t-1}+x-\xi\right) \mid \mathcal{F}_{t}\right] \mid \mathcal{F}_{t-1}\right] .
$$

The local gradient is clearly bounded by

$$
\left(\left|\Delta S_{t-1}\right|+1\right) \mathbb{E}\left[l^{\prime}\left(-H+\sum_{\ell=t+1}^{N} \theta_{\ell}^{*} \cdot \Delta S_{\ell}+\theta \cdot \Delta S_{t-1}+x-\xi\right) \mid \mathcal{F}_{t}\right]
$$

so that, using assumption $2.2(i)$, this quantity is bounded by

$$
\begin{aligned}
C\left(\left|\Delta S_{t-1}\right|+1\right) \mathbb{E}\left[l\left(-H+\sum_{\ell=t+1}^{N} \theta_{\ell}^{*} \cdot \Delta S_{\ell}+\theta \cdot \Delta S_{t-1}+x-\xi\right) \mid \mathcal{F}_{t}\right] \\
\leq C\left(\left|\Delta S_{t-1}\right|+1\right) \mathbb{E}\left[l\left(-H+\sum_{\ell=t+1}^{N} \tilde{\theta}_{\ell} \cdot \Delta S_{\ell}+\theta \cdot \Delta S_{t-1}+x-\xi\right) \mid \mathcal{F}_{t}\right] .
\end{aligned}
$$

Consequently, in order to justify the local differentiation under (conditional) expectation, we prove that for every $R>0$, the family of random variables

$$
\left(\left(\left|\Delta S_{t-1}\right|+1\right) \mathbb{E}\left[l\left(-H+\sum_{\ell=t+1}^{N} \tilde{\theta}_{\ell} \cdot \Delta S_{\ell}+\theta \cdot \Delta S_{t-1}+x-\xi\right) \mid \mathcal{F}_{t}\right] ;(x, \theta) \in \bar{B}_{1}(0, R) \times \bar{B}_{d}(0, R)\right)
$$


is (conditinally) uniformly integrable. Actually, it uses assumptions 2.2 (ii), (iii) and similar arguments as those used in Step 1. Hence, the function $G_{t-1}$ is continuously differentiable and we have

$$
\begin{aligned}
\nabla_{\theta} G_{t-1}\left(V_{t-2}^{\theta}, \theta_{t-1}\right) & =\mathbb{E}\left[\Delta S_{t-1} \mathbb{E}\left[l^{\prime}\left(-H+\sum_{\ell=t+1}^{N} \theta_{\ell}^{*} \cdot \Delta S_{\ell}+\theta_{t-1} \cdot \Delta S_{t-1}+V_{t-2}^{\theta}-\xi\right) \mid \mathcal{F}_{t}\right] \mid \mathcal{F}_{t-1}\right] \\
& =\mathbb{E}\left[\Delta S_{t-1} l^{\prime}\left(-H+\sum_{\ell=t+1}^{N} \theta_{\ell}^{*} \cdot \Delta S_{\ell}+\theta_{t-1} \cdot \Delta S_{t-1}+V_{t-2}^{\theta}-\xi\right) \mid \mathcal{F}_{t-1}\right],
\end{aligned}
$$

and,

$$
\begin{aligned}
\nabla_{x} G_{t-1}\left(V_{t-2}^{\theta}, \theta_{t-1}\right) & =\mathbb{E}\left[\mathbb{E}\left[l^{\prime}\left(-H+\sum_{\ell=t+1}^{N} \theta_{\ell}^{*} \cdot \Delta S_{\ell}+\theta_{t-1} \cdot \Delta S_{t-1}+V_{t-2}^{\theta}-\xi\right) \mid \mathcal{F}_{t}\right] \mid \mathcal{F}_{t-1}\right] \\
& =\mathbb{E}\left[l^{\prime}\left(-H+\sum_{\ell=t+1}^{N} \theta_{\ell}^{*} \cdot \Delta S_{\ell}+\theta_{t-1} \cdot \Delta S_{t-1}+V_{t-2}^{\theta}-\xi\right) \mid \mathcal{F}_{t-1}\right],
\end{aligned}
$$

so that, $\theta_{t-1}^{*}$ satisfies

$$
\mathbb{E}\left[\Delta S_{t-1} l^{\prime}\left(-H+\sum_{\ell=t+1}^{N} \theta_{\ell}^{*} . \Delta S_{\ell}+\theta_{t-1}^{*} \cdot \Delta S_{t-1}+V_{t-2}^{\theta}-\xi\right) \mid \mathcal{F}_{t-1}\right]=0 \text { a.s. }
$$

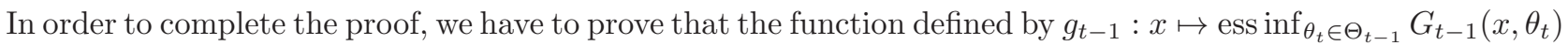
is differentiable. This is again the envelope theorem. It follows easily using similar arguments as those used in the end of step 1 . 